\title{
Light-triggered and phosphorylation-dependent 14- 3-3 association with NON-PHOTOTROPIC HYPOCOTYL 3 is required for hypocotyl phototropism
}

\section{Lea Reuter}

ZMBP- University of Tuebingen

\section{Tanja Schmidt}

ZMBP- University of Tuebingen https://orcid.org/0000-0001-9203-8847

\section{Prabha Manishankar}

ZMBP- University of Tuebingen

\section{Christian Throm}

ZMBP- University of Tuebingen https://orcid.org/0000-0003-2914-7025

\section{Jutta Keicher}

ZMBP- University of Tuebingen

\section{Andrea Bock}

ZMBP- University of Tuebingen

Claudia Oecking ( $\nabla$ claudia.oecking@zmbp.uni-tuebingen.de)

ZMBP- University of Tuebingen

\section{Article}

Keywords: Auxin-dependent Phototrophic Growth Response, Polyacidic Phospholipids, Plasma Membrame Association, NPH3 Dephosphorylation

Posted Date: May 4th, 2021

DOI: https://doi.org/10.21203/rs.3.rs-467981/v1

License: (c) (1) This work is licensed under a Creative Commons Attribution 4.0 International License. Read Full License

Version of Record: A version of this preprint was published at Nature Communications on October 21st, 2021. See the published version at https://doi.org/10.1038/s41467-021-26332-6. 
1 Light-triggered and phosphorylation-dependent 14-3-3 association with

2 NON-PHOTOTROPIC HYPOCOTYL 3 is required for hypocotyl phototropism

3

4 Lea Reuter", Tanja Schmidt\#, Prabha Manishankar, Christian Throm, Jutta Keicher, Andrea

5 Bock, Claudia Oecking*

6

7 Center for Plant Molecular Biology (ZMBP), Plant Physiology, University of Tübingen,

8 Germany

9

10 " Authors contributed equally

11 * Corresponding author. Email: claudia.oecking@zmbp.uni-tuebingen.de 
NON-PHOTOTROPIC HYPOCOTYL 3 (NPH3) is a key component of the auxin-dependent plant phototropic growth response. We show that NPH3 directly binds polyacidic phospholipids, required for plasma membrane association in darkness. We further demonstrate that blue light induces an immediate phosphorylation of a C-terminal 14-3-3 binding motif in NPH3. Subsequent association of 14-3-3 proteins is causal for the lightinduced release of NPH3 from the membrane and required for NPH3 dephosphorylation. In the cytosol, NPH3 dynamically transitions into membrane-less condensate-like structures. The dephosphorylated state of the 14-3-3 binding site and NPH3 membrane recruitment are recoverable in darkness. NPH3 variants that constitutively localize either to the membrane or to condensates are non-functional, revealing a fundamental role of the 14-3-3 mediated dynamic change in NPH3 localization for auxin-dependent phototropism. This novel mechanism of regulation might be of general nature, given that several members of the $\mathrm{NPH} 3-$ like family interact with $14-3-3$ via a C-terminal motif.

\section{Introduction}

Developmental plasticity of plants is impressively demonstrated by the phototropic response, through which plants align their growth with incoming blue light $(B L)^{1}$. Shoots typically grow towards the light by generating a lateral gradient of the growth promoting phytohormone auxin. Here, the hormone concentration is higher on the shaded side as compared with the lit side, resulting in differential growth. It is well established that the phototropins phot 1 and phot2 function as primary photoreceptors controlling phototropism in Arabidopsis ${ }^{2,3,4}$. Phototropins are plasma membrane (PM)-associated, light-activated protein kinases and indeed, BL-induced autophosphorylation turned out to be a primary and essential step for the asymmetric growth response ${ }^{5}$. In this context, members of the 14-3-3 family were identified as phot1 interactors in Arabidopsis. Eukaryotic 14-3-3 proteins are known to interact with a multitude of polypeptides in a phosphorylation-dependent manner, thereby regulating distinct cellular processes ${ }^{6}$. Plant 14-3-3 are crucial components regulating auxin transport-related development and polarity of PIN-FORMED (PIN) auxin efflux carriers ${ }^{7}$. As yet, however, a functional role of phot1/14-3-3 association could not be proven ${ }^{5,8}$. Furthermore, evidence for trans-phosphorylation activity of phototropins is surprisingly limited.

The polar localization of PIN proteins within the PM made them likely candidates promoting formation of the auxin gradient that precedes phototropic growth ${ }^{9}$. Indeed, a mutant lacking the three major PINs expressed in aerial plant parts (PIN3, PIN4, PIN7) is severely compromised in phototropism ${ }^{10}$. Notably, unilateral illumination polarizes PIN3 specifically to the inner lateral side of hypocotyl endodermis cells, aligning PIN3 polarity with the light direction and presumably redirecting auxin flow towards the shaded side ${ }^{11}$. Moreover, the 
activity of PINs is positively regulated by two protein kinase families from the AGCVIII class, namely PINOID and D6 PROTEIN KINASES ${ }^{12}$. Though phototropins belong to the same kinase class, direct PIN phosphorylation could not be demonstrated ${ }^{11}$. Taken together, signaling events that couple photoreceptor activation to changes in PIN polarization and consequently auxin relocation remain mainly elusive. In this regard, the PM-associated NON-PHOTOTROPIC HYPOCOTYL 3 (NPH3) might represent a promising component of early phototropic signaling events. It acts downstream of the photoreceptors and appears to be instrumental for auxin redistribution ${ }^{3,4,13,14}$. NPH3 possesses - in addition to the central NPH3 domain - two putative protein-protein interaction domains, a C-terminal coiled-coil (CC) domain and a N-terminal BTB/POZ (b-broad-complex, tramtrack, bric a brac/Pox virus and żinc finger) domain ${ }^{1,15}$ (Fig. S1). Indeed, NPH3 physically interacts not only with the photoreceptor phot1 but also with further early signaling elements, such as ROOT PHOTOTROPISM (RPT2) ${ }^{16}$ - another member of the plantspecific NPH3/RPT2-like family (NRL) - and defined members of the PHYTOCHROME KINASE (PKS) family ${ }^{17,} 18$. Interestingly, NPH3 exists in a phosphorylated form in dark-grown seedlings and becomes rapidly dephosphorylated upon phot1 activation ${ }^{19}, 20$. Later on, the alteration in phosphorylation status was shown to correlate closely with light-driven changes in the subcellular localization of NPH3 which detaches from the PM upon irradiation, forming aggregated particles in the cytosol ${ }^{21}$. As found for light-triggered dephosphorylation ${ }^{19}$, formation of the NPH3 particles is reversible upon darkness or prolonged irradiation ${ }^{21}$. One factor required for the recovery of phosphorylated NPH3 at the PM over periods of prolonged irradiation is its interaction partner RPT2 ${ }^{21}$. Altogether, this has led to the current model that the phosphorylation status of NPH3 determines its subcellular localization and function: phosphorylation of NPH3 promotes its action in mediating phototropic signaling from the PM, whereas NPH3 dephosphorylation reduces it by internalizing NPH3 into aggregates 4, 13, 21, 22 . As yet, however, the functional significance of NPH3 (de)phosphorylation remains poorly understood $^{20,23}$.

Here, we identified members of the 14-3-3 family as novel interactors and major regulators of $\mathrm{NPH} 3$. Our analyses revealed that BL induces phosphorylation of the antepenultimate NPH3 residue which in turn enables 14-3-3 association. Complex formation interferes with the ability of NPH3 to bind to polyacidic phospholipids, resulting in its displacement from the PM. Accumulation of NPH3 in the cytosol causes formation of membrane-less condensates. Intriguingly, both PM association and 14-3-3 triggered PM dissociation are required for NPH3 function. Taking the reversibility of the light-induced processes into account, the phototropintriggered and 14-3-3-mediated dynamic change in the subcellular localization of NPH3 seems to be crucial for its proper function in the phototropic response. 


\section{Results}

\section{PM association of NPH3 is phospholipid-dependent and requires its C-terminal} domain Association of the hydrophilic NPH3 with the PM is known since its discovery in $1999{ }^{1}$. As yet, the molecular mechanism of NPH3 membrane recruitment in darkness remains elusive. MACCHI-BOU 4 (MAB4)/ ENHANCER OF PINOID (ENP), another member of the NRL family, was recently shown to associate with the PM in a PIN-dependent manner ${ }^{24}$. Besides protein-protein interactions, hydrophobic as well as protein-lipid interactions can cause membrane anchoring of proteins. Several members of the AGCVIII kinase family - though not phot1- contain a basic and hydrophobic $(\mathrm{BH})$ motif in the middle domain of the kinase. This polybasic motif interacts directly with phospholipids and is required for PM binding ${ }^{25}$. When we applied the $\mathrm{BH}$ score prediction ${ }^{26}$ to $\mathrm{NPH} 3$, two putative $\mathrm{BH}$ motifs were identified in its C-terminal domain (Fig. S2B). To examine the importance of electronegativity for NPH3 PM association in the dark, we made use of a genetic system that depletes the polyacidic phosphoinositide (PI) phosphatidylinositol-4-phosphate (PI4P) at the PM via lipid anchoring of the catalytic domain of the yeast SAC1 PI4P phosphatase ${ }^{27,28}$. Transient co-expression of $\mathrm{NPH} 3$ together with SAC1, but not the catalytically inactive version SAC1DEAD, displaced $\mathrm{NPH} 3$ from the PM into discrete cytosolic bodies in darkness (Fig. 1A), reminiscent of the aggregated particles that have been observed upon $B L$ treatment ${ }^{21,22}$. The strong and unique electrostatic signature of the plant PM is powered by the additive effect of PI4P and the phospholipids phosphatidic acid (PA) and phosphatidylserine (PS) ${ }^{28,29,30,31}$. In lipid overlay assays, NPH3 bound to several phospholipids characterized by polyacidic headgroups, namely PA as well as the PIs PI3P, PI4P, PI5P, $\mathrm{PI}(3,4) \mathrm{P}_{2}, \mathrm{PI}(3,5) \mathrm{P}_{2}, \mathrm{PI}(4,5) \mathrm{P}_{2}$ and $\mathrm{PI}(3,4,5) \mathrm{P}_{3}$ (Fig. 1B). NPH3 did neither bind to phospholipids with monoacidic headgroups, such as phosphatidylinositol or PS, nor to phospholipids with neutral headgroups, namely phosphatidylcholine (PC) and phosphatidylethanolamine (PE). Deletion of the C-terminal 51 residues of NPH3 (NPH3 $\triangle \mathrm{C} 51$, still comprising the CC domain, Fig. S1) abolished lipid binding, while the bacterially expressed C-terminal 51 residues of NPH3 (NPH3-C51) turned out to be sufficient to bind to polyacidic phospholipids (Fig. 1B). Moreover, NPH3-C51 bound to large unilamellar liposomes containing the polyacidic phospholipids PI4P or PA, but not to liposomes composed of only neutral phospholipids such as PC and PE (Fig. 1C). Apparently, the C-terminal 51 residues of NPH3 enable electrostatic association with membrane bilayers irrespective of posttranslational protein modifications or association with other proteins. As expected, transient expression of GFP:NPH3 $\Delta \mathrm{C} 51$ in $N$. benthamiana (native or $35 \mathrm{~S}$ promoter) revealed loss of PM recruitment in the dark, as evident by the presence of discrete bodies in the cytosol (Fig. 1D, Fig. S2A). This resembles the scenario observed upon co-expression of NPH3 and SAC1 (Fig. 1A) as well as upon 
123 transient expression of NPH3 $\triangle \mathrm{C} 65$ :GFP in guard cells of Vicia faba ${ }^{32}$. By contrast, deletion

124 of the N-terminal domain (NPH3 $\Delta \mathrm{N} 54$, still comprising the BTB domain, Fig. S1) did not affect PM association of NPH3 in darkness (Fig. 1D, Fig. S2A).

\section{An amphipathic helix is essential for phospholipid binding and PM association of}

\section{NPH3 in vivo}

As already mentioned, two polybasic motifs with a $\mathrm{BH}$ score above the critical threshold value of 0.6 (window size 11 as recommended for the detection of motifs closer to the termini, ${ }^{26}$ ) were identified in the C-terminal domain of NPH3: (i) a R-rich motif (R736-R742) close to the C-terminal tail and (ii) a K-rich motif further upstream (W700-M713) (Fig. 2A, Fig. $\mathrm{S} 2 \mathrm{~B})$. The latter is predicted to form an amphipathic helix, organized with clearly distinct positively charged and hydrophobic faces. The hydrophobic moment - a measure of the amphiphilicity - was calculated to be 0.58 (Fig. S2C), similar to the PM anchor of Remorin ${ }^{33}$. In order to test the requirement of the two motifs for membrane association, all five basic amino acids within the R-rich motif were replaced by alanine (NPH3-5KR/A). Furthermore, both hydrophobicity and positive charge of the amphipathic helix were decreased by exchange of four hydrophobic residues (NPH3-4WLM/A) and of four lysine residues (NPH3$4 \mathrm{~K} / \mathrm{A}$ ), respectively (Fig. 2A; Fig. S2B). The ability of any of the three NPH3 replacement variants to bind polyacidic phospholipids in vitro was significantly impaired (Fig. 2B, C). Nonetheless, the GFP:NPH3-5KR/A mutant remained PM-associated in the dark when transiently expressed in $N$. benthamiana (Fig. 2D). To verify that the terminal R-rich motif is dispensable for PM recruitment in vivo, NPH3 was truncated by the C-terminal 28 residues (NPH3 $\Delta$ C28). Indeed, PM anchoring was unaffected (Fig. 2D; Fig. S2D). By contrast, modification of either the amphiphilicity or the hydrophobicity of the amphipathic helix gave rise to cytosolic particle-like structures in darkness (Fig. 2D, Fig. S2D). Though these particles differ in shape and size, strict co-localization of the respective NPH3 variants was observed upon co-expression (Fig. S2E). Taken together, these experiments revealed the necessity of the amphipathic helix for PM anchoring in vivo and indicate hydrophobic interactions to also contribute to PM-association of NPH3. Thus, one attractive hypothesis is

152 that the positively charged residues interact electrostatically with polyacidic phospholipids of 153 the PM followed by partial membrane penetration. By this means, interactions with both the 154 polar headgroups and the hydrocarbon region of the bilayer would be established in darkness, causing anchor properties of NPH3 similar to intrinsic proteins. 

manner

158 A yeast two hybrid screen performed in our lab (see $\left.{ }^{34}\right)$ identified NPH3 as putative interactor of several Arabidopsis 14-3-3 isoforms, among those epsilon and omega (Fig. 3A). 14-3-3 mediated regulation of NPH3 might thus represent an early event in phototropic signaling. Complex formation of NPH3 and 14-3-3 was confirmed in planta by co-immunoprecipitation (ColP) of fluorophore-tagged proteins transiently co-expressed in $N$. benthamiana leaves (Fig. 3B). To elucidate the impact of light on 14-3-3/NPH3 complex assembly, transgenic Arabidopsis lines expressing 14-3-3 epsilon:GFP under control of the native promoter ${ }^{7}$ and, as control, UBQ10::GFP were employed. Three-days old etiolated seedlings were either maintained in complete darkness or irradiated with $\mathrm{BL}\left(1 \mu \mathrm{mol} \mathrm{m} \mathrm{m}^{-2} \mathrm{sec}^{-1}\right)$ for 30 minutes. Potential targets of 14-3-3 epsilon:GFP were identified by stringent ColP-experiments coupled with mass spectrometry (MS)-based protein identification. As expected, several known 14-3-3 clients ${ }^{7}$ were detected by MS, and remarkably, NPH3 emerged as a major 143-3 interactor (Table S1). Binding capability of characterized 14-3-3 targets, such as the $\mathrm{H}^{+}-$ ATPase (AHA1) and cytosolic invertase 1 (CINV1), was not modified by BL treatment. By contrast, NPH3 turned out to be a BL-dependent 14-3-3 interactor in planta (Fig. 3C, Table S1). ColP of fluorophore-tagged proteins transiently co-expressed in $N$. benthamiana leaves confirmed that physical association of NPH3 and 14-3-3 is not detectable in darkness while BL irradiation triggers complex formation (Fig. 3D). Assuming 14-3-3 association to depend on phosphorylation of the target protein, this observation is in apparent contrast to the lightinduced dephosphorylation of NPH3 ${ }^{19}$.

178 The specific phosphorylatable 14-3-3 binding sequences of numerous target proteins are 179 most flexible and disordered ${ }^{35}$. Since both the $\mathrm{N}$ - and $\mathrm{C}$-terminal domain of NPH3 are predicted to be intrinsically disordered (Fig. S1, ${ }^{36}$ ), the corresponding truncated versions were analyzed by yeast two hybrid assays. While NPH3 $\Delta$ N54 was capable of 14-3-3 binding, deletion of the C-terminal 51 residues (NPH3 $\Delta \mathrm{C} 51$ ) abolished 14-3-3 association, suggesting that the 14-3-3 binding site - in addition to the membrane targeting motif- localizes downstream of the $\mathrm{CC}$ domain (Fig. 3A). We therefore exchanged amino acid residues, phosphorylation of which has recently been demonstrated in planta (S722, S723, S744, $S 746,{ }^{37,38}$ ), for a non-phosphorylatable alanine. Strikingly, 14-3-3 binding was not affected in all but one NPH3 mutant: replacement of S744 - the antepenultimate residue of NPH3 prevented 14-3-3 association both in yeast (Fig. 3A) and in planta (Fig. 3B), suggesting a phosphorylation-dependent C-terminal 14-3-3 binding motif (pS/pTX 1-2- $\left.^{-C O O H}\right)^{39}$ in NPH3. 

14-3-3 association is required for NPH3 function and its BL-induced PM dissociation To address the issue of functional significance of 14-3-3 association in vivo, GFP-tagged $\mathrm{NPH} 3$ variants were expressed in a T-DNA induced loss of function allele of NPH3, nph3-7 ${ }^{41}$. GFP:NPH3 was fully functional in restoring the severe impairment of hypocotyl phototropism in nph3-7, regardless of whether expression was driven by the native or the 35S CaMV promoter (Fig. 4A, Fig. S3A), thus confirming previous data ${ }^{21,22}$. By contrast, phototropic hypocotyl bending was still significantly reduced when NPH3 incapable of 14-3-3 association (GFP:NPH3-S744A) was expressed (Fig. 4A, Fig. S3A), indicating that BLinduced interaction with 14-3-3 is required for proper NPH3 function.

Though NPH3 is hydrophilic in nature, both GFP:NPH3 and GFP:NPH3-S744A localized to the cell periphery in the hypocotyl of etiolated transgenic seedlings (Fig. 4B, Fig. S3B),

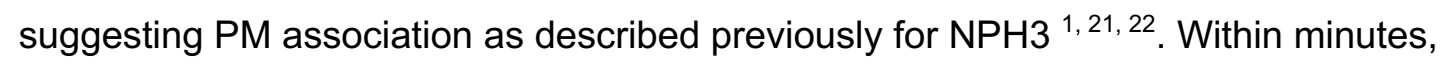
however, the BL laser used to excite GFP (488 $\mathrm{nm}$, activates phototropins), induced detachment of NPH3 from the PM into discrete bodies/particle-like structures in the cytoplasm (Video S1). This BL-induced shift in subcellular localization is mediated by phot1 activity ${ }^{21}$ and again, could be observed independent of whether expression of NPH3 was under control of the endogenous (Fig. S3B; ${ }^{22}$ ) or the $35 \mathrm{~S}$ promoter (Fig. 4B, ${ }^{21}$ ). By contrast, GFP:NPH3-S744A remained mainly PM-associated upon irradiation (Fig. 4B, Video S2, Fig. S3B, C). Mutation of the 14-3-3 binding site does thus not affect PM association of NPH3 in darkness but prevents BL-triggered PM dissociation, suggesting that light-induced binding of 14-3-3 proteins to the antepenultimate, presumably phosphorylated residue $S 744$ is required to internalize NPH3 from the PM into cytosolic particles. Nonetheless, the suspected phosphorylation of S744 might per se decrease the interaction of NPH3 with polyacidic phospholipids, hence triggering PM dissociation. Yet, the appropriate phosphomimic version of NPH3 (NPH3-S744D) was neither impaired in phospholipid-interaction in vitro (Fig. 1B) nor PM recruitment in vivo (Fig. 1D). Altogether, the C-terminal domain plays a dual role in determining the subcellular localization of NPH3: it mediates phospholipid-dependent PM association and allows for PM dissociation as a result of 14-3-3 association.

We confirmed our findings in transiently transformed N. benthamiana leaves (Fig. 4C, D; Videos S3, S4). Here, primarily RFP-tagged proteins were employed since excitation of RFP $(558 \mathrm{~nm})$ - unlike GFP (488 nm) - does not activate phototropins. This enabled us to conditionally activate phot1 by means of the GFP laser. It became evident that NPH3 instead of being directly internalized into discrete bodies - initially detaches from the PM and moves along cytoplasmic strands comparable to soluble polypeptides (Video S3). Body

$$
\text { formation in the cytosol is initiated after a lag time of approximately } 4 \text { to } 5 \text { minutes. }
$$

Generation of particle-like structures might thus depend on soluble NPH3 exceeding a critical 
concentration in the cytosol. Upon co-expression of GFP-tagged 14-3-3s, colocalization with NPH3 was observed in such particles (Fig. S3D).

\section{NPH3 forms membrane-less condensates in the cytosol}

$233 \mathrm{BL}$-induced PM dissociation and particle assembly of NPH3 in the cytosol seem to be separate and consecutive processes (Video S3). As yet, the identity of these particles has not been determined. NPH3 $\triangle \mathrm{C} 51$ is devoid of the amphipathic helix and localized to cytosolic particles in darkness (Fig. 1D). Subcellular fractionation clearly illustrated that the lack of the C-terminal region shifts NPH3 from a membrane-associated state to the soluble fraction (Fig. 1E). This reveals a non-membrane-attached state of $\mathrm{NPH} 3$ in discrete bodies as has been suggested for NPH3 aggregates generated upon BL irradiation ${ }^{21}$. Apparently, the mechanisms of NPH3 targeting towards and away from the PM are distinct from vesiclemediated transport of transmembrane proteins. This is in line with the observation that NPH3 is insensitive to an inhibitor of endosomal trafficking ${ }^{21}$. Considering the lack of the 14-3-3 binding motif in NPH3 $\triangle \mathrm{C} 51$ 1, 14-3-3 association seems dispensable for NPH3 body formation in the cytosol. To confirm this assumption, we examined NPH3 variants incapable of 14-3-3 binding, namely (i) NPH3-4K/A-S744A and (ii) NPH3-S744A, the latter upon co-expression with SAC1. Indeed, prevention of 14-3-3 association did not affect assembly of NPH3-4K/AS744A particles in darkness (Fig. S2D). Similar to NPH3, NPH3-S744A localized to cytosolic particles in the dark upon co-expression of SAC1 but not SAC1DEAD (Fig. 1A). Generation of $\mathrm{NPH} 3$ particles is hence feasible in the absence of 14-3-3s and might be due to intrinsic properties of NPH3 when exceeding a critical concentration in the cytosol. Taking constitutive PM association of NPH3-S744A in the absence of SAC1 into account, 14-3-3 association seems to be crucial for initial PM detachment while formation of discrete bodies in the cytosol occurs as an autonomous process.

254 The dynamic generation and morphology of NPH3 bodies is reminiscent of membrane-less biomolecular condensates which are micron-scale compartments in cells lacking surrounding membranes. An important organizing principle is liquid-liquid phase separation driven by multivalent macromolecular interactions - either mediated by modular interaction domains or disordered regions ${ }^{42}$. NPH3 is characterized by both intrinsically disordered regions and interaction domains such as the BTB and the CC domain (Fig. S1). We performed single-cell

260 time-lapse imaging of RFP:NPH3 body formation to investigate whether NPH3 undergoes 261 transition from a solute to a condensed state in $N$. benthamiana. Indeed, formation of 262 particle-like structures in the cytosol is initiated after approx. $4 \mathrm{~min}$ and the fluorescence 263 intensity per body gradually increased over time as a result of the growth in size (Fig. 4E, F). 264 In contrast to the signal intensity, the number of bodies reached a maximum after approx. 10 
to $15 \mathrm{~min}$ and afterwards started to decrease as a result of body fusion (Fig. 4E, G). Worth mentioning, these features are characteristic criteria of biomolecular condensates ${ }^{42,43}$.

Phosphorylation of the 14-3-3 binding site in NPH3 is light-dependent and reversible

In dark-grown seedlings, NPH3 exists as a phosphorylated protein irrespective of phot1 activity. Light-induced dephosphorylation of NPH3 is almost a dogma in the literature. It has been recognized as a slight shift in electrophoretic mobility of NPH3 upon SDS-PAGE ${ }^{19}$ and requires - in accordance with the light-induced formation of particle-like structures in the cytosol ${ }^{21}$ - the photoreceptor phot1. In the following, (de)phosphorylation of NPH3, represented by a modification of its electrophoretic mobility, will be referred to as 'general' (de)phosphorylation of NPH3. Nonetheless, the data presented so far suggest that lighttriggered and presumably S744 phosphorylation-dependent 14-3-3 association contributes to $\mathrm{NPH} 3$ function - an obvious antagonism to the 'dogma of dephosphorylation'. A phosphositespecific peptide antibody ( $\alpha$-pS744) was therefore established (antigen: ${ }_{734}$ PPRKPRRWRN$\left.\mathrm{S}(\mathrm{P})-\mathrm{IS}_{746}\right)$ and an antibody against the unmodified peptide ( $\left.\alpha-\mathrm{NPH} 3\right)$ served as control. Examination of GFP:NPH3 in either $N$. benthamiana leaves or transgenic Arabidopsis lines revealed the typical enhanced electrophoretic mobility upon BL excitation (Fig. 5), indicative of a 'general' dephosphorylation ${ }^{19,20,21}$. Intriguingly, the $\alpha$-pS744 antibody recognized GFP:NPH3, but not GFP:NPH3-S744A, exclusively upon BL irradiation (Fig. 5). BL hence triggers two different posttranslational modifications of NPH3: (i) the phosphorylation of the 14-3-3 binding site (S744) and (ii) a 'general' dephosphorylation. Yet, neither of the modifications could be observed for GFP:NPH3-S744A (Fig. 5A). To uncover light-induced 14-3-3 association at the molecular level, an IP of GFP:NPH3 was conducted and combined with 14-3-3 Far Western analysis. Phosphorylation of S744 indeed enabled binding of purified recombinant 14-3-3 proteins to NPH3 upon SDS PAGE (Fig. 5A, B). Prolonged irradiation or transfer of BL-irradiated seedlings to darkness is known to confer PM reassociation of $\mathrm{NPH} 3^{21}$, correlating with a reduced electrophoretic mobility, indicative of a 'general' re-phosphorylation ${ }^{19,}{ }^{21}$. Remarkably, we observed simultaneous dephosphorylation of S744 (Fig. 5B, C), effectively preventing binding of 14-3-3 to NPH3 (Fig. 5B). Taken together, the dark/light-dependent phosphorylation status of S744 determines 14-3-3 association with NPH3. In addition, the phosphorylation status of the 14-3-3 binding site and of NPH3 'in general' is modulated by the light regime in an opposite manner, giving rise to a coinciding, but inverse pattern. Time course analyses, however, proved S744 phosphorylation of NPH3 to precede 'general' dephosphorylation upon BL treatment (Fig. $5 \mathrm{C}$ ). 'General' dephosphorylation of NPH3 has been assumed to determine PM release of $\mathrm{NPH} 3$ coupled to particle assembly in the cytosol $4,13,21,22$. Our data now clearly indicate 
not of condensate assembly in the cytosol. 'General' dephosphorylation might thus be coupled to PM dissociation and/or condensate formation. We examined the 'general' phosphorylation status of both NPH3 and NPH3-S744A when co-expressed with SAC1. Despite the fact that either NPH3 variant constitutively localized to cytosolic condensates (Fig. 1A), NPH3 was phosphorylated in darkness and shifted to the dephosphorylated status upon BL treatment, while NPH3-S744A exhibited a permanent phosphorylated state (Fig. 5D). 'General' dephosphorylation of NPH3 is thus not coupled to PM dissociation. Moreover, it is neither a prerequisite nor a consequence of condensate assembly, rather it seems to require prior light-triggered and S744 phosphorylation-dependent 14-3-3 association (Fig. $5 A, D)$. Taken together, we suggest (Fig. 6E) that BL-induced and phosphorylationdependent 14-3-3 association releases NPH3 from the PM into the cytosol and very likely provokes 'general' dephosphorylation of NPH3. Formation of NPH3 condensates is, however, determined by the biological properties of PM-detached NPH3.

\section{Cycling of NPH3 might be key to function}

The light-triggered and reversible shift in subcellular localization of NPH3 has led to the hypothesis that PM localization of NPH3 promotes its action in mediating phototropic signaling. In turn, NPH3 present in soluble condensates is considered to be inactive ${ }^{13,21,22}$.

The functional relevance of the transient changes in subcellular NPH3 localization is, however, still not known. To assess the functionality of NPH3 variants constitutively localizing to condensates, GFP:NPH3-4K/A (Fig. 2D) as well as GFP:NPH3 $\Delta$ C51 (Fig. 1D) were expressed in the loss of function Arabidopsis mutant nph3-7. Worth mentioning, the electrophoretic mobility of GFP:NPH3-4K/A corresponded to the dephosphorylated version of $\mathrm{NPH} 3$ and was not modified by light treatment (Fig. 6C). In line with the hypothesis mentioned above, NPH3 mutants constitutively present in condensates did not restore hypocotyl phototropism (Fig. 6A, B, Videos S5, S6). Contrary to the hypothesis, however, GFP:NPH3-S744A - despite exhibiting constitutive PM localization (Fig. 4B) - is also largely incapable of mediating phototropic hypocotyl bending in nph3-7 (Fig. 4A). To verify significantly impaired activity of permanently PM-attached NPH3, we examined NPH3 $\triangle \mathrm{C} 28$ in addition. Comparable to the results obtained in N. benthamiana (Fig. 2D, Fig. S2D), $\mathrm{NPH} 3 \Delta \mathrm{C} 28$ remained PM-associated upon activation of phot1 in stable transgenic Arabidopsis lines (Fig. 6B, Video S7) and its electrophoretic mobility was not modified by BL treatment (Fig. $6 \mathrm{C}$ ). Noteworthy, both NPH3-S744A and NPH3 $\Delta \mathrm{C} 28$ still interacted with phot 1 (Fig. 6D), indicating that complex formation at the PM is not compromised. Nevertheless, permanent attachment of NPH3 to the PM turned out to be insufficient for triggering the phototropic response in nph3-7 (Fig. 6A). 
Taken together, neither NPH3 mutants permanently detached from the PM nor NPH3 versions permanently attached to the PM seem to be fully functional (Fig. 6A, E). So, what is the underlying mechanism of NPH3 function? We examined NPH3 $\Delta$ N54 (Fig. 1D, Fig. S2A, Video S8) in more detail. Similar to NPH3, NPH3 $\Delta$ N54 associated to the PM in etiolated seedlings (Fig. 6B). Upon irradiation it (i) became phosphorylated at S744 (Fig. 6C), (ii) exhibited an increased electrophoretic mobility, indicative of a 'general' dephosphorylation (Fig. 6C) and (iii) detached from the PM followed by condensate formation in the cytosol (Fig. 6B, Video S9). Furthermore, all these processes were reverted when seedlings were retransferred to darkness (Fig. 6B, C). Intriguingly, expression of NPH3 $\Delta$ N54 completely restored phototropic hypocotyl bending in nph3-7 (Fig. 6A) as did NPH3 (Fig. 4A). Thus, 143-3 mediated cycling of NPH3 between the PM and the cytosol might be of utmost importance for functionality (Fig. 6E).

\section{Discussion}

Our data provide novel insight into the molecular mechanisms defining NPH3 function in BLinduced phototropic hypocotyl bending. We applied a combination of genetic, biochemical, physiological and live cell imaging approaches to uncover the impact of 14-3-3 proteins on $\mathrm{NPH} 3$, in particular its BL-triggered, phosphorylation-dependent and functionally essential release from the PM. Association of NPH3 with the PM is known since decades, but how it is recruited to this compartment is unknown. We demonstrated that NPH3 attaches to the PM in a phospholipid-dependent manner in darkness (Fig. 1A). The electrostatic interaction with polyacidic phospholipids (Fig. 1B, C) is mediated by four basic residues of an amphipathic helix, the hydrophobic face of which further contributes to PM association (Fig. 2D). We therefore suggest the amphipathic helix to be embedded in the PM inner-leaflet with its hydrophobic interface inserted in the hydrophobic core of the bilayer while the positively charged interface is arranged on the PM surface, interacting with the lipid polar heads. The molecular mechanism underlying PM association of NPH3 is thus different from the NRL protein MAB4/ENP which is recruited to the PM by interaction with PIN proteins ${ }^{24}$. The amphipathic helix of NPH3 (amino acids 700-713) localizes downstream of the CC domain of $\mathrm{NPH} 3$ in its C-terminal region which also encompasses the 14-3-3 binding site (S744) (Fig. 2A).

We discovered that $\mathrm{BL}$ induces two distinct posttranslational modifications in NPH3 (Fig. 5):

370 (i) the immediate phosphorylation of S744 which in turn enables association of 14-3-3

371 proteins with NPH3, followed by (ii) the well-described dephosphorylation, represented by an 372 enhanced electrophoretic mobility of NPH3 ('general' dephosphorylation) ${ }^{19,20,21}$. The - as yet unrecognized - BL-induced NPH3 phosphorylation event linked to 14-3-3 association is of utmost importance since it is essential for (i) the BL-triggered internalization of NPH3 from 
the PM (Fig. 4B) and (ii) the function of NPH3 in phototropic hypocotyl bending (Fig. 4A). However, expression of NPH3-S744A which is incapable of 14-3-3 interaction, partially restored the severe impairment of hypocotyl phototropism in nph3-7 (Fig. 4A). This might be due to functional redundancy among certain members of the NRL protein family. Indeed, RPT2 is required for hypocotyl phototropism at light intensities utilized in our assays ${ }^{21}$ and its expression is induced and stabilized by BL treatment ${ }^{44}$. RPT2 might thus partially substitute for NPH3. The same applies to DEFECTIVELY ORGANIZED TRIBUTARIES 3 (DOT3), the, as yet, functionally uncharacterized closest homolog of $\mathrm{NPH} 3{ }^{13}$. Worth mentioning, RPT2, DOT3 and also MAB4/ENP are capable of interacting with 14-3-3 proteins in yeast (Fig. S4). In each case, exchange of the antepenultimate residue (serine) abolished 14-3-3 association (Fig. S4), suggesting that phosphorylation-dependent $14-3-3$ binding is not limited to NPH3 but rather represents a more widespread mechanism of NRL regulation. However, residual activity of NPH3-S744A in phototropic hypocotyl bending might alternatively be caused by its permanent association with the PM. Light treatment could induce a reorganization of NPH3-S744A within/along the PM which might allow for phototropic responsiveness to a certain level. Addressing these alternatives represents a formidable challenge for future research.

$392 \mathrm{NPH} 3$ has been described to re-localize directly from the PM into discrete bodies in the cytosol upon light treatment ${ }^{21,22}$. It became, however, evident that it initially detaches from the PM into the cytosol (Video S3). Here, NPH3 undergoes a dynamic transition from a dilute to a condensed state, resulting in the formation of membrane-less biomolecular compartments (Fig. 1E; Fig. 4E). Biomolecular condensates are emerging as an important concept in signaling, also in plants ${ }^{45}$. Their formation can be driven by multivalent interactions with other macromolecules, by intrinsically disordered regions within a single molecule or both ${ }^{42,46}$. Interestingly, 14-3-3 proteins are dispensable for condensate assembly in the cytosol, as demonstrated by 14-3-3 binding-deficient NPH3 variants (Fig. 1A,

401 D; Fig. S2D). Further studies will reveal whether condensate formation of the PM-detached $402 \mathrm{NPH} 3$ is essential for its action.

403 As described above, the light-triggered modifications of the phosphorylation pattern of NPH3 404 are highly complex. Our observations disproved the view that BL-triggered 'general' dephosphorylation events determine PM dissociation of $\mathrm{NPH}^{13,21,22}{ }^{2}$. First of all, dephosphorylation of NPH3 - i.e. a decrease in negative charge - is entirely inappropriate to interfere with membrane association relying on electrostatic interactions with polyacidic phospholipids. Furthermore, investigation of the seven NPH3 phosphorylation sites that were recently identified in etiolated Arabidopsis seedlings revealed that the phosphorylation status of these NPH3 residues was neither required for PM association in darkness nor BL-induced 
site in NPH3 (S744A) abolished PM dissociation upon BL treatment (Fig. 4B-D), indicating

413 light-induced and phosphorylation-dependent 14-3-3 association to mediate PM release of

414 NPH3. Given that the amphipathic helix localizes approximately $30-45$ residues upstream

415 of the 14-3-3 binding site (Fig. 2A), 14-3-3 binding to NPH3 is expected to induce a

416 substantial conformational change that liberates the amphipathic helix from the PM. The

417 molecular mechanism of NPH3 internalization is hence different from the - likewise PM-

418 associated - photoreceptor phot1, trafficking of which occurs via vesicles through the

419 endosomal recycling pathway ${ }^{47}$. Now, what about the BL-triggered 'general'

420 dephosphorylation of NPH3? Based on our findings, this posttranslational modification

421 temporally succeeded light-induced S744 phosphorylation (Fig. 5C). Furthermore, 'general'

422 dephosphorylation was coupled to BL-triggered S744 phosphorylation, irrespective of the

423 subcellular localization of NPH3 (Fig. 5A, D). We therefore assume phosphorylation-

424 dependent 14-3-3 binding to be required for BL-induced 'general' dephosphorylation of NPH3

425 as well - a hypothesis that will be examined by future research.

426 Re-transfer of BL-irradiated seedlings to darkness triggers (i) dephosphorylation of S744

427 linked to 14-3-3 dissociation. 14-3-3 release is expected to result in a (re)exposure of the

428 amphipathic helix, which subsequently enables (ii) re-association with the PM and

429 presumably (iii) re-phosphorylation of $\mathrm{NPH} 3$, represented by a reduced electrophoretic

430 mobility ('general' re-phosphorylation) (Fig. 5B, C). Intriguingly, neither NPH3 variants that

431 constitutively localize to the PM nor mutant versions constitutively detached from the PM are

432 capable of restoring the severe defect in hypocotyl phototropism in nph3-7. Complementation

433 of the nph3-7 phenotype exclusively could be observed upon expression of NPH3 variants

434 that exhibit a light regime-driven dynamic change in subcellular localization (Fig. 6A, B, C). In

435 summary, we propose a model where S744 phosphorylation-dependent and 14-3-3 driven

436 cycling of NPH3 between the PM and the cytosol critically determine NPH3 function in

437 mediating phototropic signaling in Arabidopsis (Fig. 6E).

438 In the past, it has been hypothesized that the light-induced internalization of phot1 - first

439 described in $2002^{48}$ - may be coupled to light-triggered re-localization of auxin transporters.

440 Functionality of phot1, however, was unaffected when internalization of the photoreceptor

441 was effectively prevented by PM tethering via lipid anchoring ${ }^{49}$. Altogether, the change in

442 subcellular localization does not seem to be essential for signaling of phot1, but of its

443 downstream signaling component NPH3 (Fig. 6E). Light-induced and 14-3-3-mediated

444 detachment of NPH3 from the PM might hence account for BL-driven changes in PIN polarity

445 required for hypocotyl phototropism. Plant 14-3-3 proteins have been shown to contribute to

446 the subcellular polar localization of PIN auxin efflux carrier and consequently auxin transport-

447 dependent growth ${ }^{7}$. NRL proteins in turn act as signal transducers in processes involving

448 auxin (re)distribution in response to developmental or environmental signals ${ }^{13}$, hence 
providing a likely link between 14-3-3 and PIN polarity. One subfamily of the NRL protein family consists of MAB4/ ENP-like (MEL) polypeptides, playing a critical role in auxinregulated organogenesis in Arabidopsis ${ }^{50,51,52}$. MEL proteins exhibited a polar localization at the cell periphery which was almost identical to that of PIN proteins ${ }^{53,54}$ and were recently shown to maintain PIN polarity by limiting lateral diffusion ${ }^{24}$. Thus, one attractive hypothesis is that certain NRL proteins contribute either to the maintenance or to a dynamic change of the subcellular polarity of PIN auxin carriers, thereby regulating auxin (re)distribution. Given that several NRL proteins are able to interact with 14-3-3 via a C-terminal binding motif (Fig. S4), phosphorylation-dependent 14-3-3 association might constitute a crucial mechanism of regulation for NRL proteins and consequently polarity of PIN proteins.

\section{Material and Methods}

\section{1}

\section{Plant materials, transformation and growth conditions}

Arabidopsis thaliana (ecotype Columbia-0 (Col-0)) expressing 14-3-3 epsilon:GFP under control of the native promoter has been described recently ${ }^{7}$. Seeds of $A$. thaliana nph3-7 (SALK_110039, Col-0 background) were obtained from the Nottingham Arabidopsis Stock Centre. T-DNA insertion was confirmed by genomic PCR analysis and homozygous lines were identified. Stable transformation of $n p h 3-7$ followed standard procedures. medium ( $\mathrm{pH}$ 5.8). Following stratification in the dark for $48-72 \mathrm{~h}$ at $4^{\circ} \mathrm{C}$, seeds were exposed to fluorescent white light for $4 \mathrm{~h}$. Subsequently, seedlings were grown at $20^{\circ} \mathrm{C}$ in darkness for $68 \mathrm{~h}$. Light treatment of etiolated seedlings was done as specified in the Figure legends. Independent experiments were carried out at least in triplicates with the same significant results. Representative images are presented. Statistics were evaluated with Excel

\section{3 (Microsoft).}

474 Transient transformation of 3-4 weeks old Nicotiana benthamiana plants was performed 475 exactly as described ${ }^{55}$. Freshly transformed tobacco plants were kept under constant light

476 for $24 \mathrm{~h}$, subsequently transferred to darkness for $17 \mathrm{~h}$ (dark adaptation) and finally irradiated

477 or kept in darkness as specified in the Figure legends.

\section{Cloning procedures}

480 A $2.1 \mathrm{~kb} N \mathrm{NH} 3$ promoter fragment was PCR-amplified from Col-0 genomic DNA and the 481 cDNA of NPH3 was amplified from Col-0 cDNA. The respective primers were characterized 482 by Bsal restriction sites allowing for the usage of the Golden Gate based modular assembly of synthetic genes for transgene expression in plants ${ }^{56}$. Following A-tailing, the individual PCR products were directly ligated into the pGEM-T Easy (Promega) vector yielding level I vectors LI A-B pNPH3 and LI C-D NPH3, respectively. Golden Gate level II assembly was 
486 performed by Bsal cut ligation and by using the modules LI A-B pNPH3, LI B-C GFP or LI B487 C mCherry, LI C-D NPH3, LI dy D-E, LI E-F nos-T and LI F-G Hygro exactly as described ${ }^{56}$. 488 For ColP of fluorophore-tagged NPH3 and 14-3-3 transiently expressed in N. benthamiana, 489 the corresponding cDNA was cloned into the 2in1 GATEWAY ${ }^{\mathrm{TM}}$ compatible vector pFRETcg$490 \quad$ 2in1-NC ${ }^{57}$ via GATEWAYTM technology.

491 Cloning of N-terminally fluorophore-tagged NPH3 variants (GFP and/or RFP) into the 492 destination vectors pB7WGR2 and/or pH7WGF2 ${ }^{58}$ for stable or transient overexpression 493 followed standard GATEWAYTM procedures. Transgenic plants were selected based on the 494 hygromycin resistance conferred by pH7WGF2 and homozygous lines were established. The 495 35S-driven PHOT1:GFP ${ }^{47}$ and the 35S::MAP:mCherry:SAC1/SAC1 $1_{\text {DEAD }}$ transformation 496 vectors ${ }^{28}$ as well as the utilized Golden Gate level I vectors ${ }^{56}$ have been described before, 497 respectively.

498 Site-directed mutagenesis was performed by PCR. PCR products and products of 499 mutagenesis were verified by sequencing.

500 A complete list of oligonucleotides used for PCR is provided below.

501

\section{Expression and purification of proteins}

503 For expression of the Arabidopsis 14-3-3 isoform omega as RGS(His) $)_{6}$-tagged protein in 504 Escherichia coli M15, the corresponding cDNA was amplified by PCR and cloned into the 505 expression vector pQE-30 (Qiagen). Purification was done by using $\mathrm{Ni}^{2+}$-NTA agarose 506 (Qiagen) according to the manufacturer's protocol.

507 For expression of the Arabidopsis NPH3 C-terminal 51 residues fused to GST in E. coli 508 BL21(DE3), the corresponding cDNA fragment was amplified by PCR and cloned into the 509 GST expression vector pGEX-4T-1. GST fusion proteins were purified from transformed 510 bacteria using GSH-Sepharose according to the manufacturer's protocol (Cytiva). Free GST 511 protein was expressed and purified as a negative control.

\section{Cell-free protein expression}

514 Reactions were performed using the TNT® T7 Quick Coupled Transcription/Translation 515 System (Promega) with $1 \mu \mathrm{g}$ of vector (NPH3 or variants in pGADT7) for a $50 \mu$ l reaction. 516 Protein expression was carried out at $30^{\circ} \mathrm{C}$ for $90 \mathrm{~min}$. Immunodetection was performed by 517 using an anti-HA antibody (HA-tag encoded by pGADT7).

\section{Preparation of microsomal membranes}

520 Microsomal membrane fractions were prepared from transiently transformed $N$. benthamiana 521 leaves. Tissue was homogenized with $3 \mathrm{~mL}$ homogenization buffer per $\mathrm{g}$ fresh weight (50 $522 \mathrm{mM}$ Hepes (pH 7.8), 500 mM sucrose, 1 \% (w/v) PVP-40, 3 mM DTT, 3 mM EDTA, 
523 supplemented with Complete Protease Inhibitor Mixture (Roche) and Phosphatase Inhibitor

524 Mix 1 (Serva)). The homogenate was centrifuged at $10,000 \mathrm{~g}$ for $20 \mathrm{~min}$ at $4{ }^{\circ} \mathrm{C}$. The

525 supernatant was filtered through MiraCloth and subsequently centrifuged at $100,000 \mathrm{~g}$ for 45

$526 \mathrm{~min}$ at $4{ }^{\circ} \mathrm{C}$. The microsomal pellet was resuspended in $5 \mathrm{mM}$ Tris/MES (pH 6.5), $330 \mathrm{mM}$

527 sucrose, 2 mM DTT, supplemented with Complete Protease Inhibitor Mixture (Roche) and

528 Phosphatase Inhibitor Mix 1 (Serva).

529

530 Phospholipid binding assays

531 For lipid binding assays, either NPH3 variants expressed in a cell free system or purified

532 recombinant GST fusion proteins were applied. Lipid overlay assays using PIP-strips were

533 performed following the manufacturer's instructions (Echelon). In brief, membranes were

534 blocked overnight at $4^{\circ} \mathrm{C}$ in a blocking buffer with $4 \%$ fatty acid-free BSA in PBS-T $(0.1 \%$

535 Tween). Purified proteins $(0.1 \mu \mathrm{g} / \mathrm{ml}$ blocking buffer) or $10-50 \mu \mathrm{l}$ of the cell free expression

536 reaction (volume adjusted according to prior immunodetection of individual reactions) were

537 incubated with PIP-strip membranes for $1 \mathrm{~h}$ at room temperature and washed three times for

53810 min with PBS-T. Subsequently, detection of bound proteins was done by

539 immunodetection of either GST (GST fusion proteins) or the HA-tag (cell free expression).

540 Liposome binding assays were conducted essentially as described by ${ }^{59}$ with slight

541 modifications. All lipids were obtained from Avanti Polar Lipids. Liposomes were prepared

542 from $400 \mathrm{nmol}$ of total lipids at the following molar ratios: PC:PE, 1:1; PC:

543 PE:PI4P, 2:2:1; PC:PE:PA, 2:2:1. The binding buffer (150 mM KCl, $25 \mathrm{mM}$ Tris-HCl pH 7.5,

$5441 \mathrm{mM}$ DTT, $0.5 \mathrm{mM}$ EDTA) was supplemented with Complete Protease Inhibitor Mixture

545 (Roche). Purified GST-NPH3-C51 variants in binding buffer were centrifuged at 50,000 $\mathrm{g}$ to

546 get rid of any possible precipitates. Following incubation of liposomes and proteins, the

547 liposome pellet was washed twice with binding buffer. Liposome-bound GST-NPH3-C51

548 variants were detected by immunoblotting with anti-GST antibodies.

549

550 Y2H, SDS-PAGE and Western Blotting

551 For yeast two-hybrid analyses, the individual constructs were cloned into the vectors

552 pGADT7 and pGBKT7 and co-transformed into the yeast strain PJ69-4A. Activity of the

553 ADE2 reporter was analyzed by growth of co-transformed yeast on SD medium lacking 554 adenine.

555 SDS-PAGE, Western blotting and immunodetection followed standard procedures. Total

556 proteins were extracted from 3-day-old etiolated Arabidopsis seedlings (50 seedlings) or

557 transiently transformed $N$. benthamiana leaves (2 leaf disks) by directly grinding in $100 \mu \mathrm{l} 2 \mathrm{x}$

558 SDS sample buffer under red safe light illumination. Chemiluminescence detection was

559 performed with an Amersham Image Quant800 (Cytiva) system. 
560 The rabbit anti-NPH3-S744P antibody was generated with the phosphorylated synthetic

561 peptide $\mathrm{NH}_{2}-\mathrm{PPRKPRRWRN}-\mathrm{S}\left(\mathrm{PO}_{3} \mathrm{H}_{2}\right)-\mathrm{IS}-\mathrm{COOH}$ followed by affinity-purifications against

562 the non-phosphorylated and phosphorylated peptide at Eurogentec (Liege, Belgium).

\section{ColP and mass spectrometry analysis}

565 Arabidopsis seedlings expressing 14-3-3 epsilon-GFP (endogenous promoter) and, as 566 control, GFP (UBQ10 promoter) were grown in the dark on half-strength MS plates for 3 567 days. Subsequently, the etiolated seedlings were either kept in darkness or treated with 568 overhead BL $\left(1 \mu \mathrm{mol} \mathrm{m}{ }^{-2} \mathrm{sec}^{-1}\right)$ for $30 \mathrm{~min}$. Three grams of plant tissue were used under red 569 safe light illumination for immunoprecipitation as described ${ }^{60}$. The final precipitate in 570 Laemmli buffer was analyzed by mass spectrometry (MS) at the University of Tübingen

571 Proteome Center. Following a tryptic in gel digestion, LC-MS/MS analysis was performed on

572 a Proxeon Easy-nLC coupled to an QExactiveHF mass spectrometer (method: 60 min,

573 Top7, HCD). Processing of the data was conducted using MaxQuant software (vs 1.5.2.8).

574 The spectra were searched against an Arabidopsis thaliana UniProt database. Raw data 575 processing was done with $1 \%$ false discovery rate setting.

576 Two individual biological replicates were performed and the following candidates were 577 omitted from the list of epsilon-GFP interaction partners: (i) proteins that interacted with GFP 578 (control), (ii) proteins that were identified in only one of the two experiments. Protein signal 579 intensities of well-known 14-3-3 client proteins (Fig. 3C) were converted to normalized 580 abundance of the bait protein. Fold changes in relative abundance of $B L$ treatment versus 581 darkness (BL vs. D) were calculated (Table S1).

582 Arabidopsis nph3-7 ectopically expressing GFP:NPH3 and N. benthamiana leaves 583 transiently overexpressing fluorophore-tagged proteins were immunoprecipitated under red 584 safe light illumination according to ${ }^{61}$. Growth and light irradiation of the plants is specified 585 elsewhere.

586 In vivo interaction of phot1:GFP and N-terminally RFP-tagged NPH3 variants was tested by 587 using solubilized microsomal proteins obtained from dark adapted $N$. benthamiana plants 588 ectopically co-expressing the proteins of interest. Solubilization was achieved by adding $5890,5 \%$ Triton X-100 to resuspended microsomal proteins followed by centrifugation at 50,000 $590 \mathrm{~g}$ for $30 \mathrm{~min}$ at $4{ }^{\circ} \mathrm{C}$. The supernatant was added to GFP-Trap Beads (ChromoTek) and 591 incubated at $4^{\circ} \mathrm{C}$ for $1 \mathrm{~h}$. Precipitated beads were washed six times with $50 \mathrm{mM}$ HEPES pH $5927.8,150 \mathrm{mM} \mathrm{NaCl}, 0,2 \%$ Triton X-100. Finally, proteins were eluted by SDS sample buffer 593 and separated by SDS-PAGE.

\section{4-3-3 Far-Western}

596 Anti-GFP immunoprecipitates obtained from Arabidopsis nph3-7 stably overexpressing 597 GFP:NPH3 were separated by SDS-PAGE and transferred to nitrocellulose. Nonspecific 
sites were blocked by incubation with $4 \%(\mathrm{w} / \mathrm{v})$ milk powder in TBS at room temperature for at least $1 \mathrm{~h}$. Subsequently, the membrane was incubated overnight at $4{ }^{\circ} \mathrm{C}$ (followed by $1 \mathrm{~h}$ at room temperature) with purified recombinant RGS(His) $)_{6}$-tagged 14-3-3 isoform omega of Arabidopsis diluted to $20 \mathrm{mg} \mathrm{ml}^{-1}$ in $50 \mathrm{mM} \mathrm{MOPS} / \mathrm{NaOH}$, pH 6.5, 20\% (w/v) glycerol, $5 \mathrm{mM}$ $\mathrm{MgCl}_{2}$, and 2mM DTT. After washing with TBS, immunodetection of RGS(His)6-tagged 14-33 was performed by applying the anti-RGS(His) 6 antibody (Qiagen) in combination with a secondary anti-mouse HRP antibody.

\section{Hypocotyl Phototropism analysis}

A. thaliana seedlings were grown in the dark on vertically oriented half-strength MS plates for $48 \mathrm{~h}$. Etiolated seedlings were then transferred to a LED chamber and illuminated with unilateral BL $\left(1 \mu \mathrm{mol} \mathrm{m} \mathrm{m}^{-2} \mathrm{sec}^{-1}\right)$ for $24 \mathrm{~h}$. Plates were scanned and the inner hypocotyl angle was measured for each seedling using Fiji. The curvature angle was calculated as the difference between $180^{\circ}$ and the measured value.

\section{Confocal microscopy}

Live-cell imaging was performed using the Leica TCS SP8 (upright) confocal laser scanning microscope. For excitation and emission of fluorophores, the following laser settings were used: GFP, excitation 488 nm, emission 505-530 nm; RFP, excitation 558 nm, emission 600$630 \mathrm{~nm}$. All CLSM images in a single experiment were captured with the same settings using the Leica Confocal Software. All the experiments were repeated at least three times. Images were processed using LAS $X$ light.

Single-cell time-lapse imaging was carried out on live leaf tissue samples from $N$. benthamiana transiently expressing RFP:NPH3. PM-detachment was induced by means of the GFP-laser (488 nM) and image acquisition (RFP-laser) was done for the duration of 32 min by scanning 30 consecutive planes along the $Z$ axis covering the entire thickness of an epidermal cell. Z-projection was done for each 3,5 min interval. For all image quantifications, randomly sampled unsaturated confocal images (512 x 512 pixels, $225 \times 225 \mu \mathrm{m})$ were used with an image analysis protocol implemented in the Image $J$ software ${ }^{62}$ as previously described ${ }^{63}$. A random image was selected from the dataset and parameters such as local threshold, background noise, object size and shape were determined. The obtained parameters were used for image analysis of the whole dataset following exactly the published step by step protocol ${ }^{63}$. 


\section{List of primers used in this study}

\begin{tabular}{|c|c|}
\hline $\begin{array}{l}\text { pGEM-T Easyl } \\
\text { yeast \& bacterial } \\
\text { expression vectors/ } \\
\text { mutagenesis }\end{array}$ & \\
\hline NPH3_Smal_F & TATcccgggCATGTGGGAATCTGAGAGCGAC \\
\hline NPH3 $\Delta$ N53_Sma_F & TATcccgggCGATCTTCTGGTTAAGATCGGC \\
\hline NPH3-C51_EcoRI_F & TATgaattcTCTTCTTCGGCTTGGACCAGC \\
\hline NPH3_Sall_R & TATgtcgacTCATGAAATTGAGTTCCT \\
\hline NPH3 $\Delta$ C51_Sall_R & TATgtcgacCTATGGCGTGTTCTTCACTTTCCC \\
\hline NPH3_S743A_Sall_R & TATgtcgacTCATGAAATTGcGTTCCTCCATCGTCT \\
\hline NPH3_S743D_Sall_R & TATgtcgacTCATGAAATgtcGTTCCTCCATCGTCTTGGTTTC \\
\hline NPH3_S743E_Sall_R & TATgtcgacTCATGAAATTtcGTTCCTCCATCGTCTTGGTTTC \\
\hline NPH3_S745A_Sall_R & TATgtcgacTCATGcAATTGAGTTCCTCCATCGTCT \\
\hline NPH3_3KR/A_Sall_R & TATgtcgacTCATGAAATTGAGTTCgcCCATgcTCTTGGTTTCgcGGGGGGTGGATGATC \\
\hline NPH3_5KR/A_Sall_R & TATgtcgacTCATGAAATTGAGTTCgcCCATgcTgcTGGTgcCgcGGGGGGTGGATGATC \\
\hline NPH3_4K/A_F & GCTTGGACCAGCGGTTGGgcGgcGCTAAGTgcACTGACTgcGATGAGTGGACAGGAGAG \\
\hline NPH3_4K/A_R & CTCTCCTGTCCACTCATCgCAGTCAGTgCACTTAGCgcCgcCCAACCGCTGGTCCAAGC \\
\hline NPH3_4WLM/A_F & TCGGCTTGGACCAGCGGTgcGAAGAAGgcAAGTAAAgcGACTAAGgcGAGTGGACAGGAGAGCCAT \\
\hline NPH3_4WLM/A_R & ATGGCTCTCCTGTCCACTCgcCTTAGTCgcTTTACTTgcCTTCTTCgcACCGCTGGTCCAAGCCGA \\
\hline NPH3_S721A_F & CAGGAGAGCCATGACATAGCCTCTGGAGGAGAACAAGCT \\
\hline NPH3_S721A_R & AGCTTGTTCTCCTCCAGAGGCTATGTCATGGCTCTCCTG \\
\hline NPH3_S722A_F & GAGAGCCATGACATATCCGCTGGAGGAGAACAAGCTGGT \\
\hline NPH3_S722A_R & ACCAGCTTGTTCTCCTCCAGCGGATATGTCATGGCTCTC \\
\hline 14-3-3omega_BamHI_F & TATggatccATGGCGTCTGGGCGTGAAGAG \\
\hline 14-3-3omega_EcoRI_F & TATgaattcATGGCGTCTGGGCGTGAAGAG \\
\hline 14-3-3omega_Sall_R & TATgtcgacTCACTGCTGTTCCTCGGT \\
\hline \multicolumn{2}{|l|}{ GATEWAY } \\
\hline NPH3_attB1_F & AAAAAGCAGGCTTAATGTGGGAATCTGAGAGCGAC \\
\hline NPH3 $\Delta$ N53_attB1_F & AAAAAGCAGGCTTAATGGATCTTCTGGTTAAGATCGGC \\
\hline NPH3_attB2_R & AGAAAGCTGGGTGTCATGAAATTGAGTTCCTCCA \\
\hline NPH3_S743A_attB2_R & AGAAAGCTGGGTGTCATGAAATTGcGTTCCTCCATCGTCT \\
\hline NPH3_S743D_attB2_R & AGAAAGCTGGGTGTCATGAAATgtcGTTCCTCCATCGTCT \\
\hline NPH3_5KR/A_attB2_R & AGAAAGCTGGGTGTCATGAAATTGAGTTCgcCCATgcTgcTGG \\
\hline NPH3 $\Delta$ C28_attB2_R & AGAAAGCTGGGTGTCAGCTCTCCTGTCCACTCATCTT \\
\hline NPH3 $\Delta$ C51_attB2_R & AGAAAGCTGGGTGCTATGGCGTGTTCTTCACTTTCCC \\
\hline NPH3_attB4_R & GAAAAGTTGGGTGTCATGAAATTGAGTTCCTCCA \\
\hline NPH3_S743A_attB4_R & GAAAAGTTGGGTGTCATGAAATTGCGTTCCTCCATCGTCT \\
\hline 14-3-3omega_attB3_F & ataataaagttgtaATGGCGTCTGGGCGT \\
\hline
\end{tabular}




\begin{tabular}{|c|c|}
\hline 14-3-3omega_attB2_R & agaaagctgggtgCTGCTGTTCCTCGGT \\
\hline attB1 adapter & GGGGACAAGTTTGTACAAAAAAGCAGGCT \\
\hline attB2 adapter & GGGGACCACTTTGTACAAGAAAGCTGGGT \\
\hline attB3 adapter & GGGGACAACTTTGTATAATAAAGTTG \\
\hline attB4 adapter & GGGGACAACTTTGTATAGAAAAGTTGGGT \\
\hline \multicolumn{2}{|l|}{ GOLDEN GATE } \\
\hline NPH3prom_A-B_F & AACAggtctcAGCGGAAACCCCACATTAATCAGACAGAATC \\
\hline NPH3prom_A-B_R & AACAggtctcACAGAACACAAGTTAACACTCTCTGTAGTTG \\
\hline NPH3_C-D_F & AACAggtctcACACCATGTGGGAATCTGAGAGCGAC \\
\hline NPH3 $\Delta$ N53_C-D_F & AACAggtctcACACCATGGATCTTCTGGTTAAGATCGGC \\
\hline NPH3_C-D_R & AACAggtctcACCTTTCATGAAATTGAGTTCCTCCA \\
\hline NPH3_S743A_C-D_R & AACAggtctcACCTTTCATGAAATTGCGTTCCTCCATCGTCT \\
\hline $\mathrm{NPH} 3 \Delta \mathrm{C} 28 \_\mathrm{C}-\mathrm{D} \_\mathrm{R}$ & AACAggtctcACCTTTCAGCTCTCCTGTCCACTCATCTT \\
\hline NPH3 $\Delta$ C51_C-D_R & AACAggtctcACCTTTCATGGCGTGTTCTTCACTTTCCC \\
\hline
\end{tabular}

\section{Acknowledgements}

634 We are grateful to Yvon Jaillais and John M. Christie for providing the constructs 35S::MAP:SAC1/SAC1 $1_{\text {DEAD }}$ and 35S::PHOT1:GFP, respectively. We furthermore thank Sandra Richter for SP8 support and John M. Christie for sharing data and stimulating discussions. MS analysis was done at the Proteome Centre, University of Tübingen, and we thank Irina Droste-Borel for help in data assessment. Research in our laboratory was supported by the German Research Foundation (DFG) with a grant to C.O. (CRC 1101B09).

\section{References}

643 1. Motchoulski A, Liscum E. Arabidopsis NPH3: A NPH1 photoreceptor-interacting protein essential for phototropism. Science 286, 961-964 (1999).

2. Christie JM, Murphy AS. Shoot phototropism in higher plants: new light through old concepts. Am J Bot 100, 35-46 (2013).

3. Fankhauser C, Christie JM. Plant phototropic growth. Current biology : CB 25, R384389 (2015).

4. Legris $M$, Boccaccini A. Stem phototropism toward blue and ultraviolet light. Physiol Plant 169, 357-368 (2020).

5. Inoue S, Kinoshita T, Matsumoto M, Nakayama KI, Doi M, Shimazaki K. Blue lightinduced autophosphorylation of phototropin is a primary step for signaling. Proc Natl Acad Sci U S A 105, 5626-5631 (2008).

6. Mackintosh C. Dynamic interactions between 14-3-3 proteins and phosphoproteins regulate diverse cellular processes. Biochem J 381, 329-342 (2004). 
7. Keicher J, et al. Arabidopsis 14-3-3 epsilon members contribute to polarity of PIN auxin carrier and auxin transport-related development. Elife 6, (2017).

8. Sullivan S, Thomson CE, Kaiserli E, Christie JM. Interaction specificity of Arabidopsis 14-3-3 proteins with phototropin receptor kinases. FEBS letters 583, 2187-2193 (2009).

9. Rakusová H, Fendrych M, Friml J. Intracellular trafficking and PIN-mediated cell polarity during tropic responses in plants. Curr Opin Plant Biol 23, 116-123 (2015).

10. Willige BC, et al. D6PK AGCVIII kinases are required for auxin transport and phototropic hypocotyl bending in Arabidopsis. Plant Cell 25, 1674-1688 (2013).

11. Ding Z, et al. Light-mediated polarization of the PIN3 auxin transporter for the phototropic response in Arabidopsis. Nat Cell Biol 13, 447-452 (2011).

12. Zourelidou M, et al. Auxin efflux by PIN-FORMED proteins is activated by two different protein kinases, D6 PROTEIN KINASE and PINOID. Elife 3, (2014).

13. Christie JM, Suetsugu N, Sullivan S, Wada M. Shining Light on the Function of NPH3/RPT2-Like Proteins in Phototropin Signaling. Plant Physiol 176, 1015-1024 (2018).

14. Hohm T, Preuten T, Fankhauser C. Phototropism: translating light into directional growth. Am J Bot 100, 47-59 (2013).

15. Pedmale UV, Celaya RB, Liscum E. Phototropism: mechanism and outcomes. Arabidopsis Book 8, e0125 (2010).

16. Inada S, Ohgishi M, Mayama T, Okada K, Sakai T. RPT2 is a signal transducer involved in phototropic response and stomatal opening by association with phototropin 1 in Arabidopsis thaliana. Plant Cell 16, 887-896 (2004).

17. de Carbonnel M, et al. The Arabidopsis PHYTOCHROME KINASE SUBSTRATE2 protein is a phototropin signaling element that regulates leaf flattening and leaf positioning. Plant Physiol 152, 1391-1405 (2010).

18. Lariguet $\mathrm{P}$, et al. PHYTOCHROME KINASE SUBSTRATE 1 is a phototropin 1 binding protein required for phototropism. Proc Natl Acad Sci U S A 103, 1013410139 (2006).

19. Pedmale UV, Liscum E. Regulation of phototropic signaling in Arabidopsis via phosphorylation state changes in the phototropin 1-interacting protein NPH3. J Biol Chem 282, 19992-20001 (2007).

20. Tsuchida-Mayama T, et al. Mapping of the phosphorylation sites on the phototropic signal transducer, NPH3. Plant Science 174, 626-633 (2008).

21. Haga K, Tsuchida-Mayama T, Yamada M, Sakai T. Arabidopsis ROOT PHOTOTROPISM2 Contributes to the Adaptation to High-Intensity Light in Phototropic Responses. Plant Cell 27, 1098-1112 (2015).

22. Sullivan S, Kharshiing E, Laird J, Sakai T, Christie JM. Deetiolation Enhances Phototropism by Modulating NON-PHOTOTROPIC HYPOCOTYL3 Phosphorylation Status. Plant Physiol 180, 1119-1131 (2019). 
23. Kimura T, Haga K, Nomura Y, Higaki T, Nakagami H, Sakai T. The Phosphorylation Status of NPH3 Affects Photosensory Adaptation During the Phototropic Response. bioRxiv https://doi.org/10.1101/2020.12.01.407205, (2020).

24. Glanc M, et al. AGC kinases and MAB4/MEL proteins maintain PIN polarity by limiting lateral diffusion in plant cells. Current biology, doi: 10.1016/j.cub.2021.02.028 (2021).

25. Barbosa IC, Shikata H, Zourelidou M, Heilmann M, Heilmann I, Schwechheimer C. Phospholipid composition and a polybasic motif determine D6 PROTEIN KINASE polar association with the plasma membrane and tropic responses. Development 143, 4687-4700 (2016).

26. Brzeska H, Guag J, Remmert K, Chacko S, Korn ED. An experimentally based computer search identifies unstructured membrane-binding sites in proteins: application to class I myosins, PAKS, and CARMIL. J Biol Chem 285, 5738-5747 (2010).

27. Gronnier J, et al. Structural basis for plant plasma membrane protein dynamics and organization into functional nanodomains. Elife 6, (2017).

28. Simon ML, et al. A Ptdlns(4)P-driven electrostatic field controls cell membrane identity and signalling in plants. Nat Plants 2, 16089 (2016).

29. Heilmann I. Phosphoinositide signaling in plant development. Development 143, 2044-2055 (2016).

30. Platre MP, et al. A Combinatorial Lipid Code Shapes the Electrostatic Landscape of Plant Endomembranes. Dev Cell 45, 465-480 e411 (2018).

31. Tejos R, et al. Bipolar Plasma Membrane Distribution of Phosphoinositides and Their Requirement for Auxin-Mediated Cell Polarity and Patterning in Arabidopsis. Plant Cell 26, 2114-2128 (2014).

32. Inoue S, Kinoshita T, Takemiya A, Doi M, Shimazaki K. Leaf positioning of Arabidopsis in response to blue light. Mol Plant 1, 15-26 (2008).

33. Perraki A, et al. Plasma membrane localization of Solanum tuberosum remorin from group 1, homolog 3 is mediated by conformational changes in a novel C-terminal anchor and required for the restriction of potato virus $\mathrm{X}$ movement]. Plant Physiol 160, 624-637 (2012).

34. Jaspert N, Throm C, Oecking C. Arabidopsis 14-3-3 proteins: fascinating and less fascinating aspects. Frontiers in Plant Science 2, 96 (2011).

35. Bustos DM, Iglesias AA. Intrinsic disorder is a key characteristic in partners that bind 14-3-3 proteins. Proteins 63, 35-42 (2006).

36. Piovesan D, et al. MobiDB: intrinsically disordered proteins in 2021. Nucleic acids research 49, D361-d367 (2021).

37. Mergner J, et al. Mass-spectrometry-based draft of the Arabidopsis proteome. Nature 579, 409-414 (2020).

38. Wang $\mathrm{P}$, et al. Mapping proteome-wide targets of protein kinases in plant stress responses. Proc Natl Acad Sci U S A 117, 3270-3280 (2020). 
39. Coblitz B, Wu M, Shikano S, Li M. C-terminal binding: an expanded repertoire and function of 14-3-3 proteins. FEBS letters 580, 1531-1535 (2006).

40. Johnson C, Crowther S, Stafford MJ, Campbell DG, Toth R, MacKintosh C. Bioinformatic and experimental survey of 14-3-3-binding sites. Biochem J 427, 69-78 (2010).

41. Kansup J, Tsugama D, Liu S, Takano T. Arabidopsis G-protein beta subunit AGB1 interacts with NPH3 and is involved in phototropism. Biochem Biophys Res Commun 445, 54-57 (2014).

42. Banani SF, Lee HO, Hyman AA, Rosen MK. Biomolecular condensates: organizers of cellular biochemistry. Nat Rev Mol Cell Biol 18, 285-298 (2017).

43. Zavaliev R, Mohan R, Chen T, Dong X. Formation of NPR1 Condensates Promotes Cell Survival during the Plant Immune Response. Cell 182, 1093-1108 e1018 (2020).

44. Kimura T, Tsuchida-Mayama T, Imai H, Okajima K, Ito K, Sakai T. Arabidopsis ROOT PHOTOTROPISM2 Is a Light-Dependent Dynamic Modulator of Phototropin1. Plant Cell 32, 2004-2019 (2020).

45. Emenecker RJ, Holehouse AS, Strader LC. Biological Phase Separation and Biomolecular Condensates in Plants. Annual review of plant biology, DOI: 10.1146/annurev-arplant-081720-015238 (2021).

46. Ruff KM, Roberts S, Chilkoti A, Pappu RV. Advances in Understanding StimulusResponsive Phase Behavior of Intrinsically Disordered Protein Polymers. Journal of molecular biology 430, 4619-4635 (2018).

47. Kaiserli E, Sullivan S, Jones MA, Feeney KA, Christie JM. Domain swapping to assess the mechanistic basis of Arabidopsis phototropin 1 receptor kinase activation and endocytosis by blue light. Plant Cell 21, 3226-3244 (2009).

48. Sakamoto K, Briggs WR. Cellular and subcellular localization of phototropin 1. Plant Cell 14, 1723-1735 (2002).

49. Preuten T, Blackwood L, Christie JM, Fankhauser C. Lipid anchoring of Arabidopsis phototropin 1 to assess the functional significance of receptor internalization: should I stay or should I go? New Phytol 206, 1038-1050 (2015).

50. Cheng Y, Qin G, Dai X, Zhao Y. NPY1, a BTB-NPH3-like protein, plays a critical role in auxin-regulated organogenesis in Arabidopsis. Proc Natl Acad Sci U S A 104, 18825-18829 (2007).

51. Furutani $\mathrm{M}$, et al. The gene MACCHI-BOU 4/ENHANCER OF PINOID encodes a $\mathrm{NPH} 3$-like protein and reveals similarities between organogenesis and phototropism at the molecular level. Development 134, 3849-3859 (2007).

52. Treml BS, et al. The gene ENHANCER OF PINOID controls cotyledon development in the Arabidopsis embryo. Development 132, 4063-4074 (2005).

53. Furutani M, Nakano $\mathrm{Y}$, Tasaka M. MAB4-induced auxin sink generates local auxin gradients in Arabidopsis organ formation. Proc Natl Acad Sci U S A 111, 1198-1203 (2014). 
54. Furutani M, et al. Polar-localized NPH3-like proteins regulate polarity and endocytosis of PIN-FORMED auxin efflux carriers. Development 138, 2069-2078 (2011).

55. Blatt MR, Grefen C. Applications of fluorescent marker proteins in plant cell biology. Methods Mol Biol 1062, 487-507 (2014).

56. Binder A, et al. A modular plasmid assembly kit for multigene expression, gene silencing and silencing rescue in plants. PLoS One 9, e88218 (2014).

57. Hecker A, Wallmeroth N, Peter S, Blatt MR, Harter K, Grefen C. Binary 2in1 Vectors Improve in Planta (Co)localization and Dynamic Protein Interaction Studies. Plant Physiol 168, 776-787 (2015).

58. Karimi M, Depicker A, Hilson P. Recombinational cloning with plant gateway vectors. Plant Physiol 145, 1144-1154 (2007).

59. Julkowska MM, Rankenberg JM, Testerink C. Liposome-binding assays to assess specificity and affinity of phospholipid-protein interactions. Methods Mol Biol 1009, 261-271 (2013).

60. Park M, Touihri S, Muller I, Mayer U, Jurgens G. Sec1/Munc18 protein stabilizes fusion-competent syntaxin for membrane fusion in Arabidopsis cytokinesis. Dev Cell 22, 989-1000 (2012).

61. Albert I, et al. An RLP23-SOBIR1-BAK1 complex mediates NLP-triggered immunity. Nature Plants 1, 15140 (2015).

62. Schindelin J, et al. Fiji: an open-source platform for biological-image analysis. Nat Methods 9, 676-682 (2012).

63. Zavaliev R, Epel BL. Imaging callose at plasmodesmata using aniline blue: quantitative confocal microscopy. Methods Mol Biol 1217, 105-119 (2015). 
A

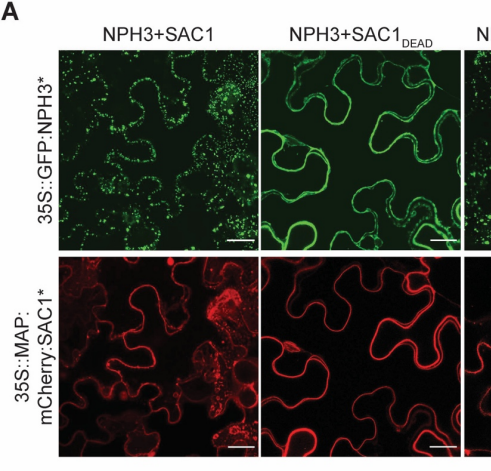

B

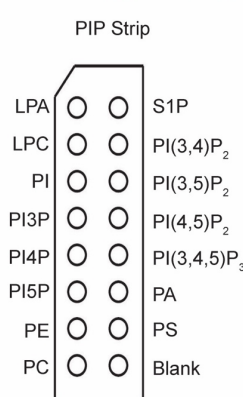

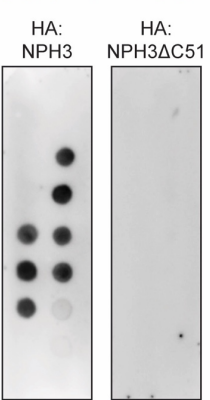

NPH3-S744A+SAC1 NPH3-S744A+SAC1
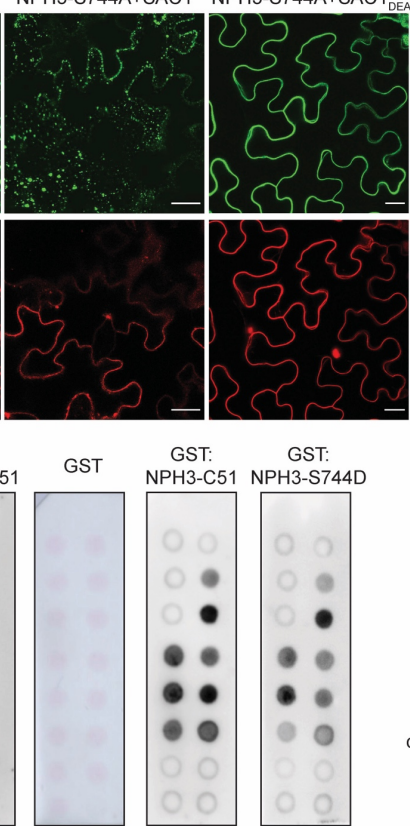

C
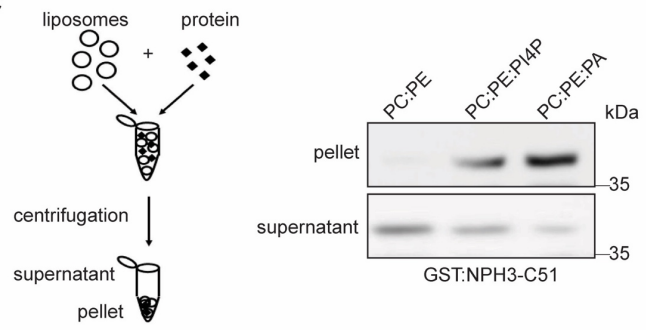

$\mathrm{NPH} 3 \Delta \mathrm{C} 51$

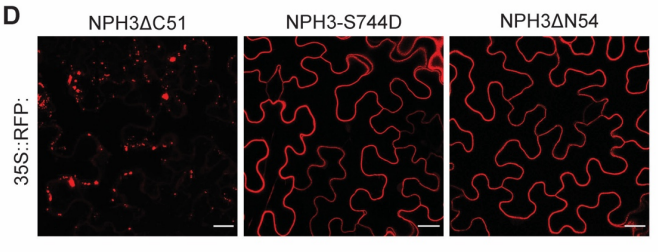

NPH3 3 N5 5

$\mathrm{E}$

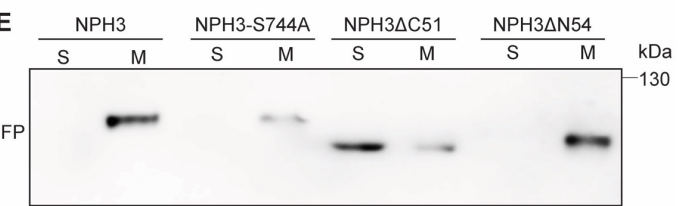

864

865

866

867

868

869

870

871

872

873

874

875

876

877

878

879

880

881

882

883

Fig. 1: NPH3 binds to polyacidic phospholipids via its C-terminal domain.

(A), (D) Representative confocal microscopy images of leaf epidermal cells from transiently transformed $N$. benthamiana adapted to darkness (Z-stack projections of NPH3 $\Delta C 51$ (D) as well as $\mathrm{NPH} 3$ variants $\left(\mathrm{NPH} 3^{*}\right)$ co-expressed with SAC1 variants $\left(\mathrm{SAC} 1^{*}\right)(\mathrm{A})$ are shown). Scale bars, $25 \mu \mathrm{m}$.

(B) Lipid overlay assay performed with either in vitro transcribed and translated HA:NPH3 and HA:NPH3 $\triangle$ C51 or purified GST and GST:NPH3-C51 variants. Immunodetection was performed by using anti-HA or anti-GST antibodies, respectively. See main text for abbreviations.

(C) Liposome binding assay using large unilamellar liposomes containing the neutral phospholipids PE and PC mixed with either the polyacidic PI4P or PA as specified. Anti-GST immunoblot of GST:NPH3-C51 is shown.

(E) Representative immunoblots with anti-GFP after subcellular fractionation of protein extracts prepared from $N$. benthamiana leaves transiently expressing 35S::GFP:NPH3 variants and adapted to darkness. Proteins in each fraction $(7.5 \mu \mathrm{g})$ were separated on $7.5 \%$ SDS-PAGE gels. Note that the total amount of soluble proteins (S) is approximately 15 times higher as compared to the total amount of microsomal proteins (M) after $100,000 \mathrm{~g}$

centrifugation. 
A

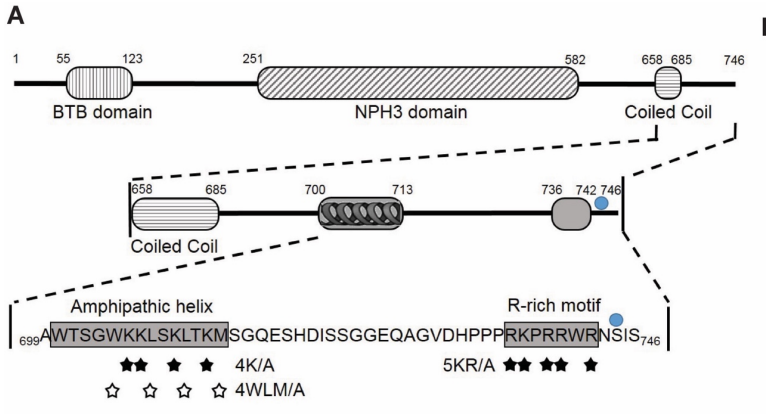$$
\text { LPA }
$$
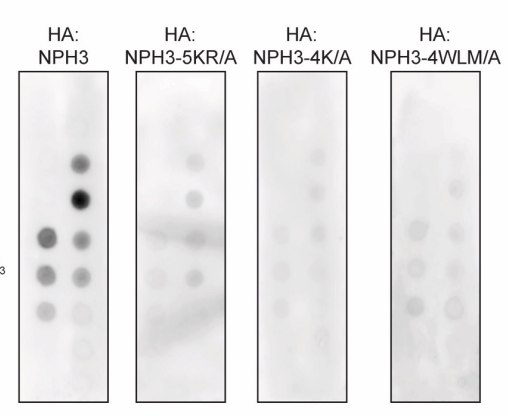

C
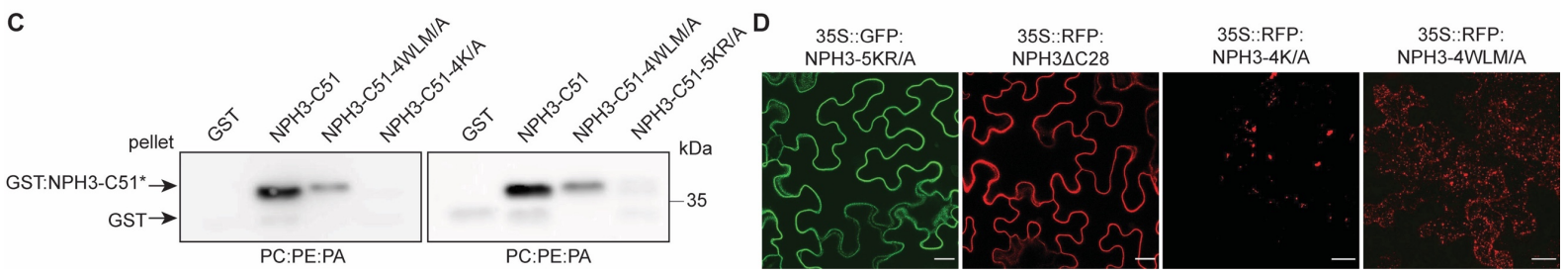

Fig. 2: An amphipathic helix within the C-terminal domain is required for NPH3 phospholipid binding, membrane association and plasma membrane localization.

(A) Domain structure and primary sequence of NPH3 showing the two putative $\mathrm{BH}$ domains (amphipathic helix and R-rich motif) within the C-terminal region. Stars depict residues of either the R-rich motif or the amphipathic helix substituted by alanine (A) in the NPH3

\section{0} variants, blue circle depicts the 14-3-3 binding site (see Fig. 3).

(B) Lipid overlay assay performed with purified GST:NPH3-C51 variants $\left(\mathrm{C} 51^{*}\right)$.

(C) Liposome binding assay using large unilamellar liposomes containing the neutral PE and PC mixed with the polyacidic PA. Anti-GST immunoblot of GST:NPH3-C51 variants is shown.

(D) Representative confocal microscopy images of leaf epidermal cells from transiently transformed $N$. benthamiana adapted to darkness (Z-stack projections of NPH3-4K/A and 
A
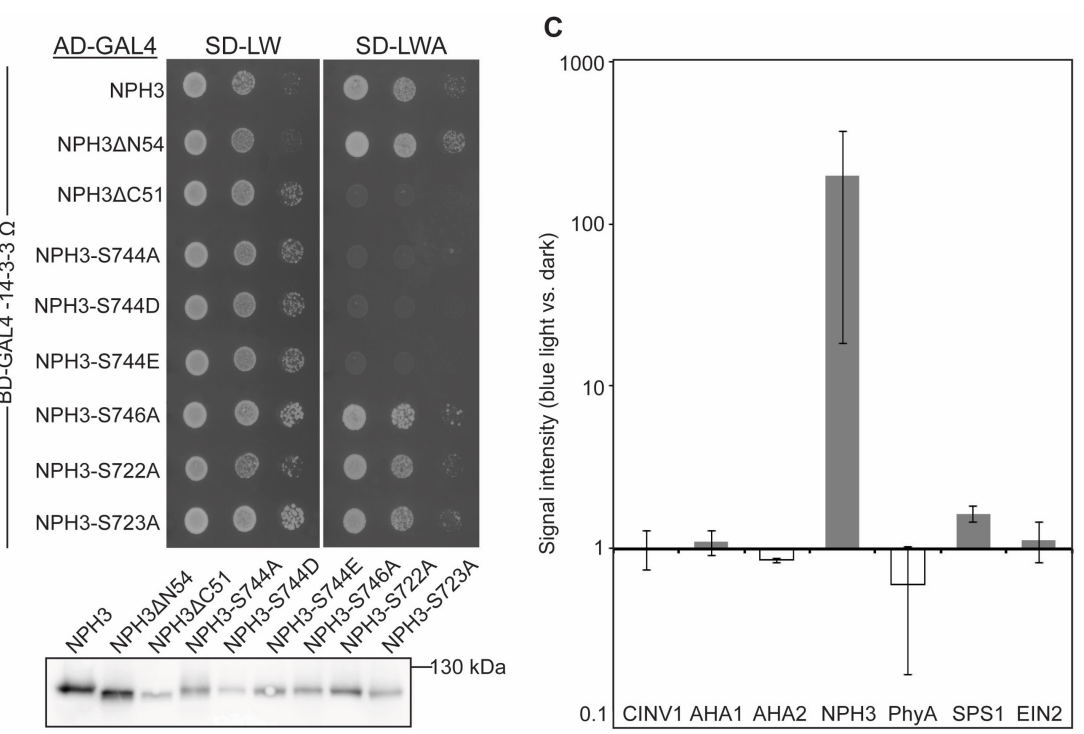

B

IP: $\alpha$-GFP
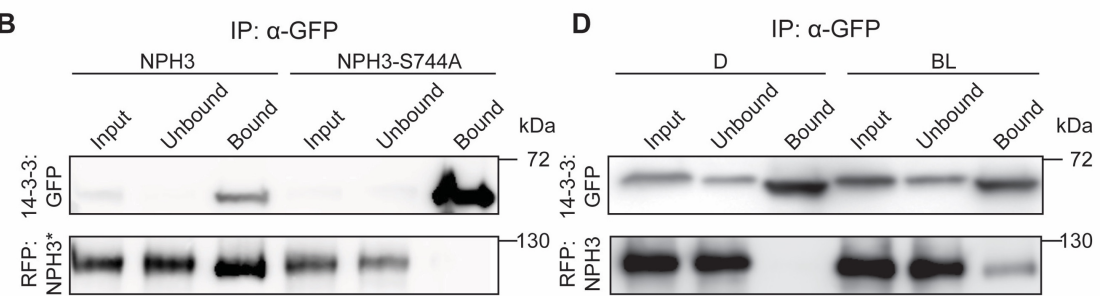

Fig. 3: Interaction of NPH3 and 14-3-3 proteins is triggered by blue light irradiation and abolished by mutation of the antepenultimate NPH 3 residue.

(A) Yeast two-hybrid interaction analysis of the Arabidopsis 14-3-3 isoform omega with NPH3 wild type and mutant variants (upper panel). Expression of the diverse NPH3 fusion proteins in yeast was confirmed by anti-HA-immunodetection (lower panel). $A D$, activating domain; $B D$, binding domain.

(B, D) In vivo interaction of mCherry:NPH3 variants and 14-3-3 omega:mEGFP in transiently transformed $N$. benthamiana leaves. Expression of transgenes was driven by the $35 \mathrm{~S}$ promoter. Freshly transformed tobacco plants were either kept under constant light for $42 \mathrm{~h}$ (B) or kept under constant light for $24 \mathrm{~h}$ and subsequently transferred to darkness for $17 \mathrm{~h}$ with (BL) or without (D) blue light treatment $\left(5 \mu \mathrm{mol} \mathrm{m}^{-2} \mathrm{sec}^{-1}\right)$ for the last 40 minutes (D). The crude extract was immunoprecipitated using GFP beads and separated on $11 \%$ SDS-PAGE gels, followed by immunoblotting with anti-GFP and anti-RFP antibodies, respectively.

(C) Arabidopsis 14-3-3 epsilon interactors were identified by mass spectrometry analysis of anti-GFP immunoprecipitations (two biological replicates) from etiolated seedlings expressing 14-3-3 epsilon:GFP either maintained in darkness or irradiated with blue light $\left(1 \mu \mathrm{mol} \mathrm{m}{ }^{-2}\right.$ $\mathrm{sec}^{-1}$ ) for $30 \mathrm{~min}$. Protein intensities of 14-3-3 client proteins were normalized to relative abundance of the bait protein (Table S1). Fold changes in relative abundance (mean $\pm S D$, logarithmic scale) of blue light treatment versus darkness are given. AHA1, AHA2, Arabidopsis $\mathrm{H}^{+}$-ATPase; CINV1, cytosolic invertase 1; EIN2, ethylene insensitive 2; PhyA, phytochrome A; SPS1, sucrose phosphate synthase 1. 
A

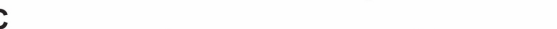

B $\quad$ D

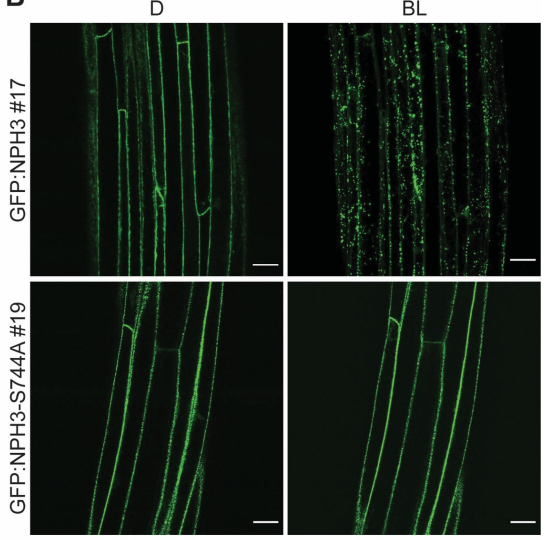

D
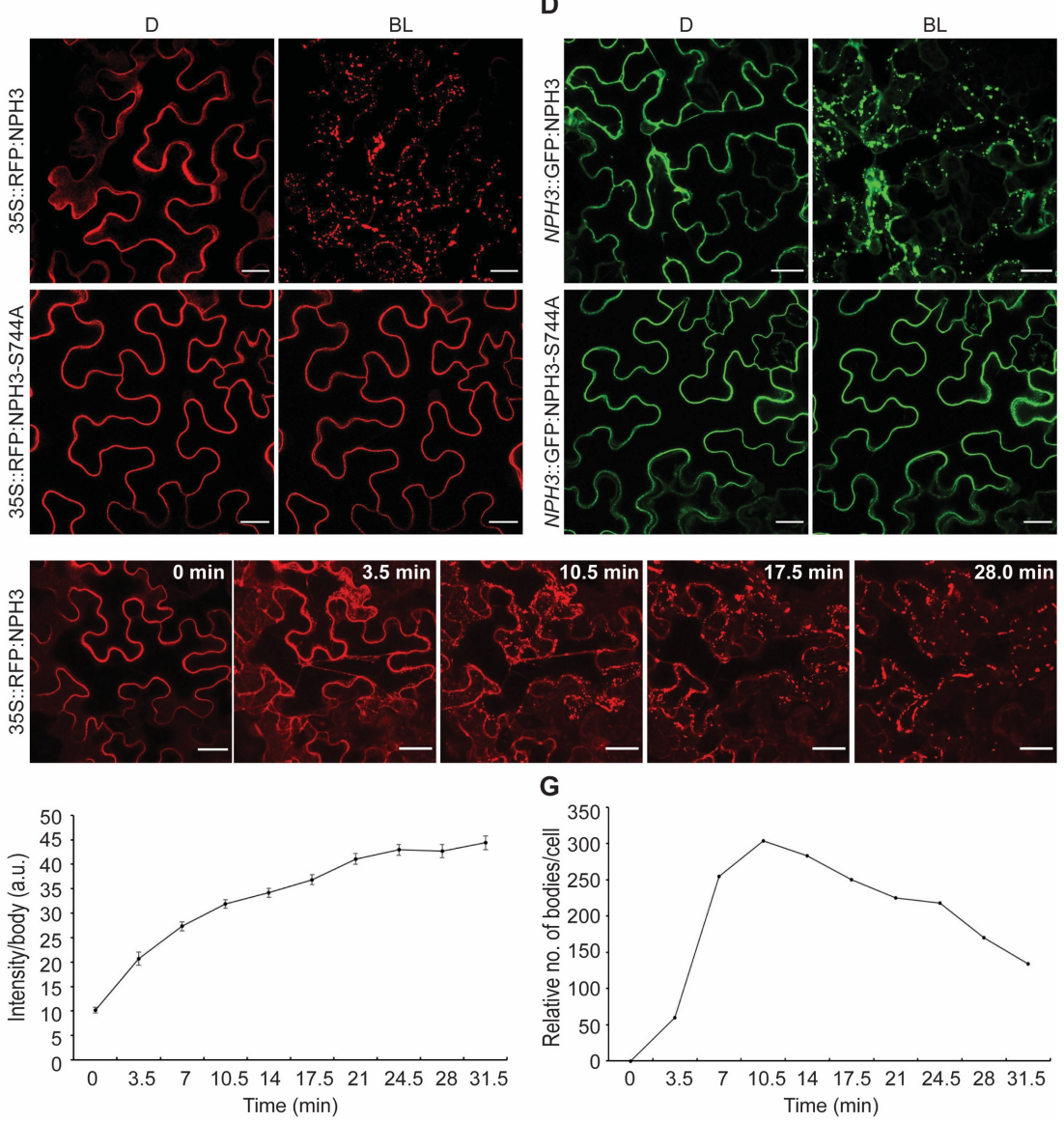

Fig. 4: 14-3-3 binding is required for proper NPH3 function in phototropic hypocotyl bending and its light-triggered detachment from the plasma membrane.

(A) Quantification of the hypocotyl phototropism response (mean \pm SEM) in 3-days old etiolated seedlings exposed for $12 \mathrm{~h}$ to unilateral blue light $\left(1 \mu \mathrm{mol} \mathrm{m}^{-2} \mathrm{sec}^{-1}\right)(\mathrm{n}>30$ seedlings per experiment, one representative experiment of two replicates is shown). Expression of transgenes in nph3-7 was driven by the 35S promoter. Student's t-test, different letters mark statistically significant differences $(P<0.05)$, same letters mark statistically non-significant differences.

(B, C, D) Representative confocal microscopy images of hypocotyl cells from transgenic etiolated Arabidopsis nph3-7 seedlings (B) or of leaf epidermal cells from transiently transformed $N$. benthamiana (Z-stack projections of BL-treated NPH3 are shown) (C, D). The plants were either kept in darkness $(D)$ or treated with blue light $(B L)(N$. benthamiana: approx. $11 \mathrm{~min}$ and $n p h 3-7$ : approx. 6 min by means of the GFP-laser). Expression of transgenes was driven by the $35 \mathrm{~S}$ promoter $(\mathbf{B}, \mathbf{C})$ or the native $N P H 3$ promoter (D). Scale bars, $25 \mu \mathrm{m}$. 
941 (E, F, G) Single-cell time-lapse imaging of RFP:NPH3 condensation induced by GFP-laser 942 treatment. The image of time point 0 image was taken in the absence of the GFP-laser. Z943 stack projections from selected time points (E), fluorescence intensity per body (mean $944 \pm$ SEM) (F) and number of bodies (G) are shown. Scale bars, $25 \mu \mathrm{m}$. 
A

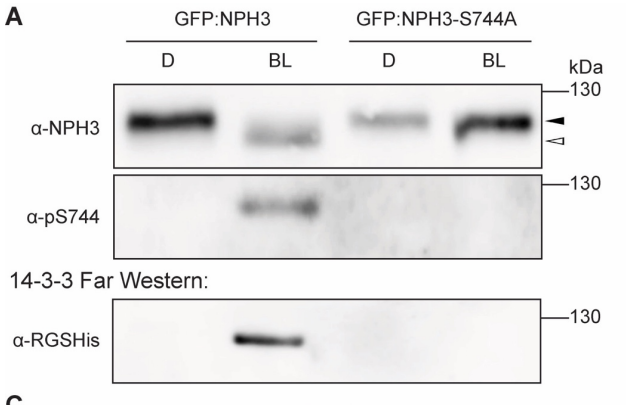

C

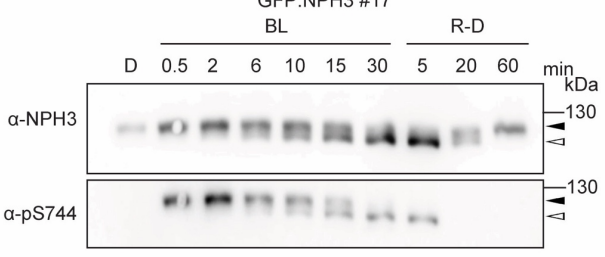

D

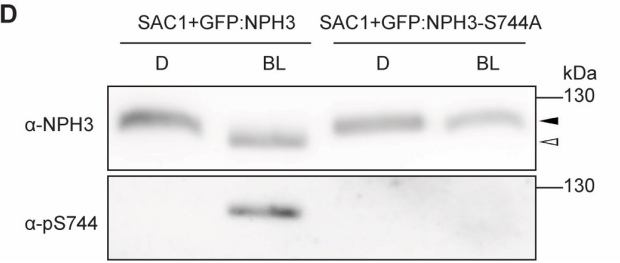

B
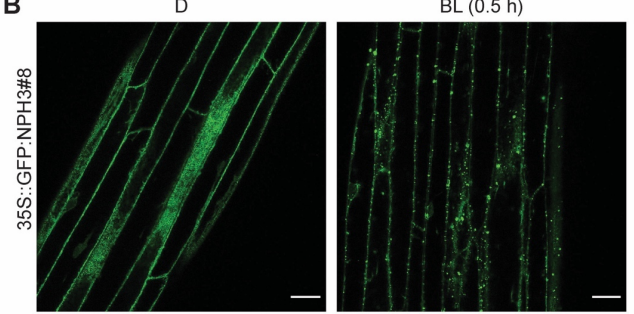

$\mathrm{BL}$
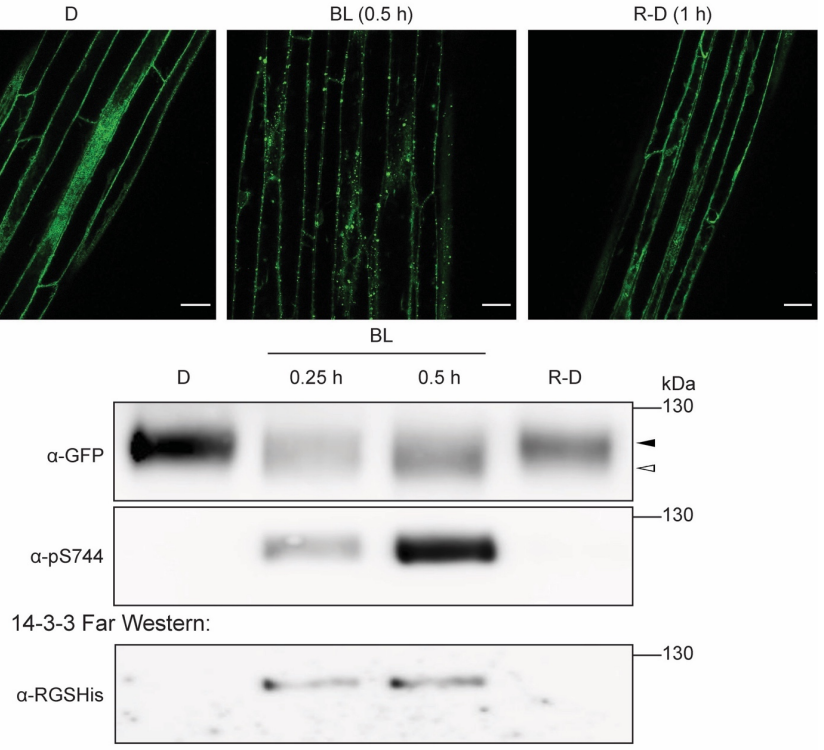

Fig. 5: The phosphorylation status of the NPH3 14-3-3 binding site is dynamically modulated by the light regime.

(A, B, C) Immunoblot analysis of total protein extracts (C) or anti-GFP immunoprecipitates and 14-3-3 Far-Western (A, B) from Arabidopsis nph3-7 ectopically expressing GFP:NPH3 or GFP:NPH3-S744A. 3-days old etiolated seedlings were treated with cycloheximide (100 $\mu \mathrm{M})$ for $1 \mathrm{~h}(\mathbf{B})$ and either maintained in darkness $(D)$, treated with blue light $(B L)\left(1 \mu \mathrm{mol} \mathrm{m}{ }^{-2}\right.$ $\left.\mathrm{sec}^{-1}\right)$ for the indicated time $(\mathbf{A}: 30 \mathrm{~min})$, or re-transferred to darkness $(1 \mathrm{~h})$ after $30 \mathrm{~min}$ of irradiation (R-D). Proteins were separated on $7.5 \%$ SDS-PAGE gels. The upper panel in (B) shows representative confocal microscopy images of hypocotyl cells from transgenic etiolated Arabidopsis seedlings under the specified conditions. Scale bars, $25 \mu \mathrm{m}$. (D) Immunoblot analysis of transiently transformed $N$. benthamiana leaves co-expressing SAC1:RFP with either GFP:NPH3 or GFP:NPH3-S744A and adapted to darkness (see Fig. 1A). Expression of transgenes was driven by the $35 S$ promoter. Total protein extracts were separated on $7.5 \%$ SDS-PAGE gels.

The closed and open arrowheads indicate the positions of 'generally' phosphorylated and dephosphorylated NPH3 proteins, respectively. 


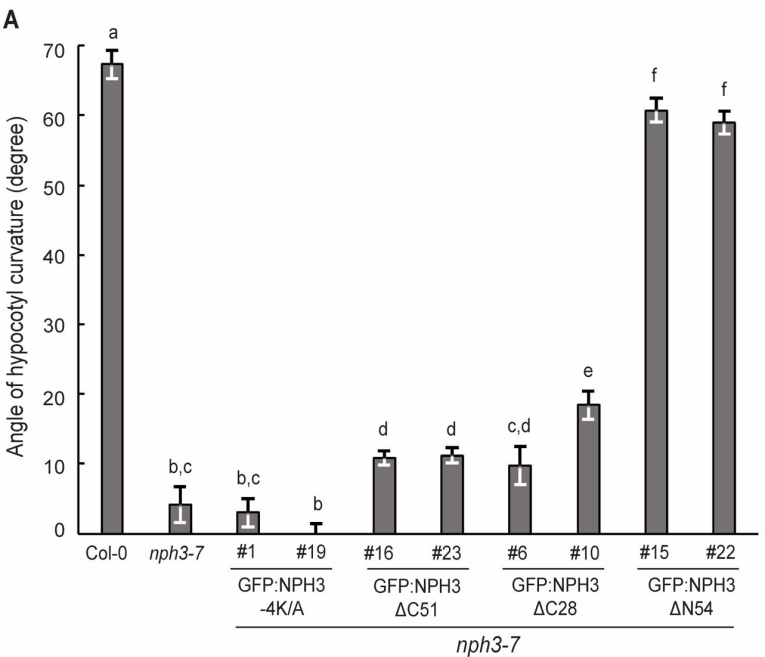

C

B
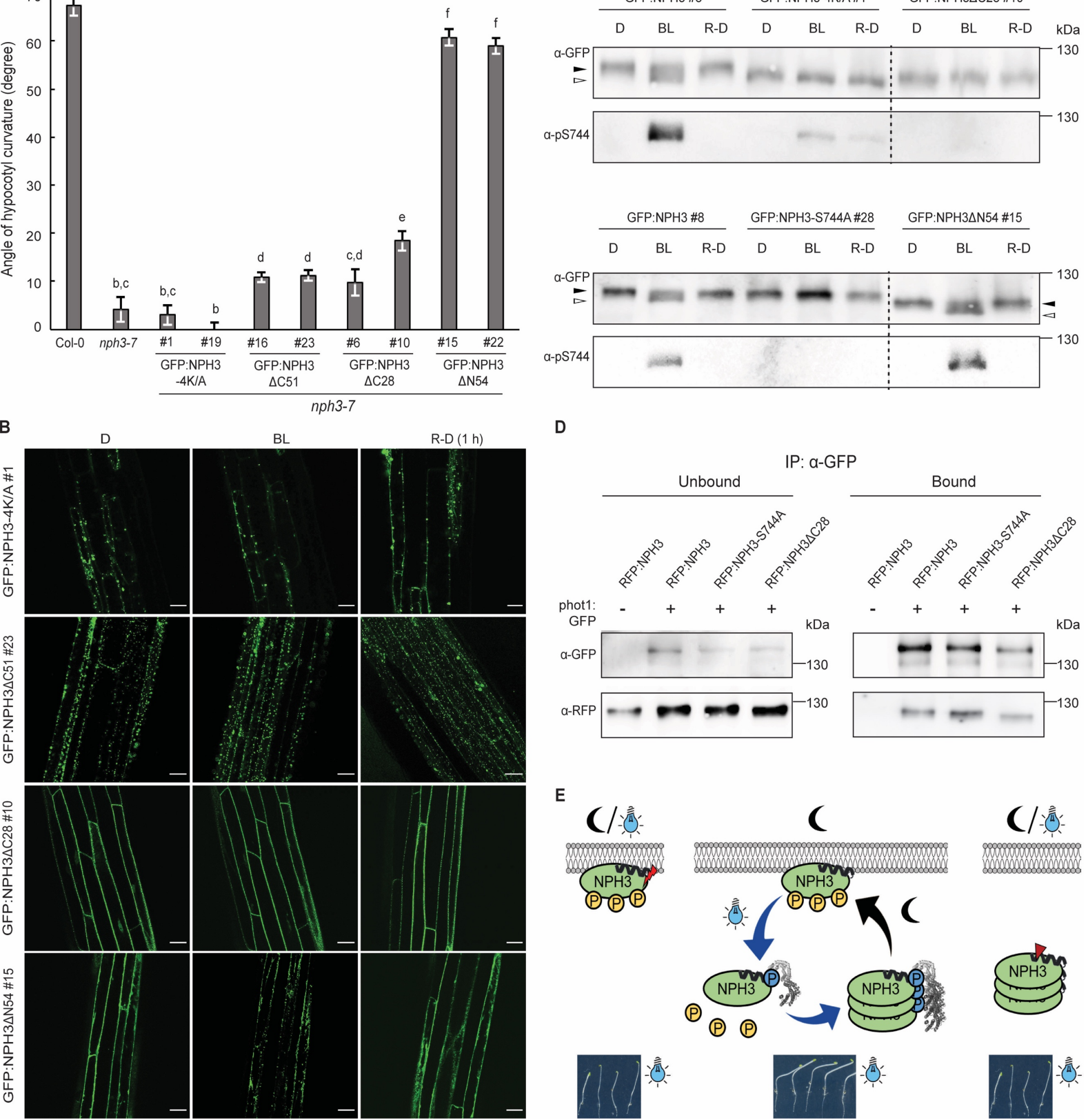

D

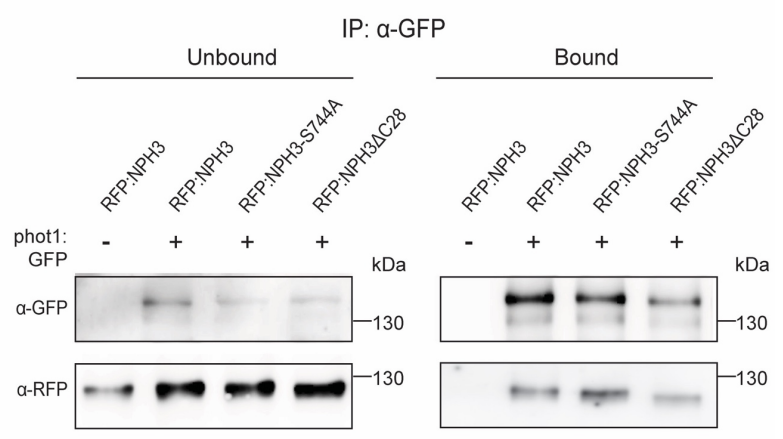

E
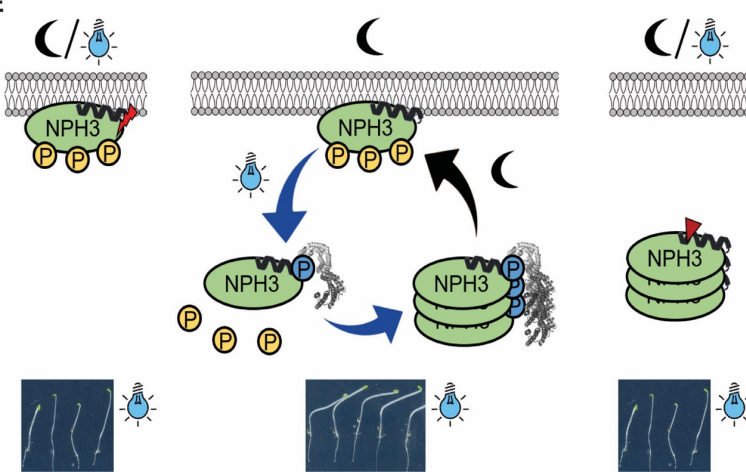

112 冷

Fig. 6: Functional relevance of the subcellular localization of NPH3.

(A) Quantification of the hypocotyl phototropism response (mean \pm SEM) in 3-days old etiolated seedlings exposed for $12 \mathrm{~h}$ to unilateral blue light $\left(1 \mu \mathrm{mol} \mathrm{m} \mathrm{mec}^{-1}\right)(\mathrm{n}>30$ seedlings per experiment, one representative experiment of two replicates is shown). Expression of wild-type and mutant variants of GFP:NPH3 in nph3-7 was driven by the 35S promoter. Student's t-test, different letters mark statistically significant differences $(P<0.05)$, same letters mark statistically non-significant differences.

(B) Representative confocal microscopy images of hypocotyl cells from transgenic Arabidopsis $n p h 3-7$ seedlings ectopically expressing mutant variants of GFP:NPH3. 3-days old etiolated seedlings were either maintained in darkness (D), treated with blue light (BL) (approx. 11 min by means of the GFP-laser) or re-transferred to darkness (1 h) (R-D) after 30 min of irradiation $\left(1 \mu \mathrm{mol} \mathrm{m} \mathrm{m}^{-2} \mathrm{sec}^{-1}\right)$. Scale bars, $25 \mu \mathrm{m}$.

(C) Immunoblot analysis of etiolated Arabidopsis nph3-7 seedlings ectopically expressing mutant variants of GFP:NPH3 and treated as described in (B). Total protein extracts were separated on $7.5 \%$ SDS-PAGE gels. All samples shown in one panel are from the same blot, the dashed line was inserted to indicate an expected modification of the molecular weight of 
$982 \mathrm{NPH} 3$ due to truncations. The closed and open arrowheads indicate the positions of 983 'generally' phosphorylated and dephosphorylated NPH3 proteins, respectively.

984 (D) In vivo interaction of RFP:NPH3 and phot1:GFP in transiently transformed $N$.

985 benthamiana leaves adapted to darkness. Expression of transgenes was driven by the 35S

986 promoter. Microsomal proteins were immunoprecipitated using GFP beads and separated on

$98711 \%$ SDS-PAGE gels, followed by immunoblotting with anti-GFP and anti-RFP antibodies,

988 respectively.

989 (E) Model depicting the light-regime triggered changes in the phosphorylation status,

990 subcellular localization and phototropic responsiveness of NPH3. BL-induced and

991 phosphorylation-dependent (S744, blue) binding of 14-3-3 proteins releases NPH3 from the

992 PM into the cytosol followed by condensate formation. Residues that are phosphorylated in

993 darkness (yellow) and become dephosphorylated upon light treatment give rise to a shift in

994 electrophoretic mobility ('general' phosphorylation status). Re-transfer to darkness reverts all

995 BL-triggered processes, finally resulting in PM re-association. Cycling of NPH3 between the

$996 \mathrm{PM}$ and the cytosol seems to be essential for proper function. Vice versa, NPH3 variants

997 either constitutively attached to (red flash) or constitutively detached (red arrowhead) from

998 the PM are non-functional. 

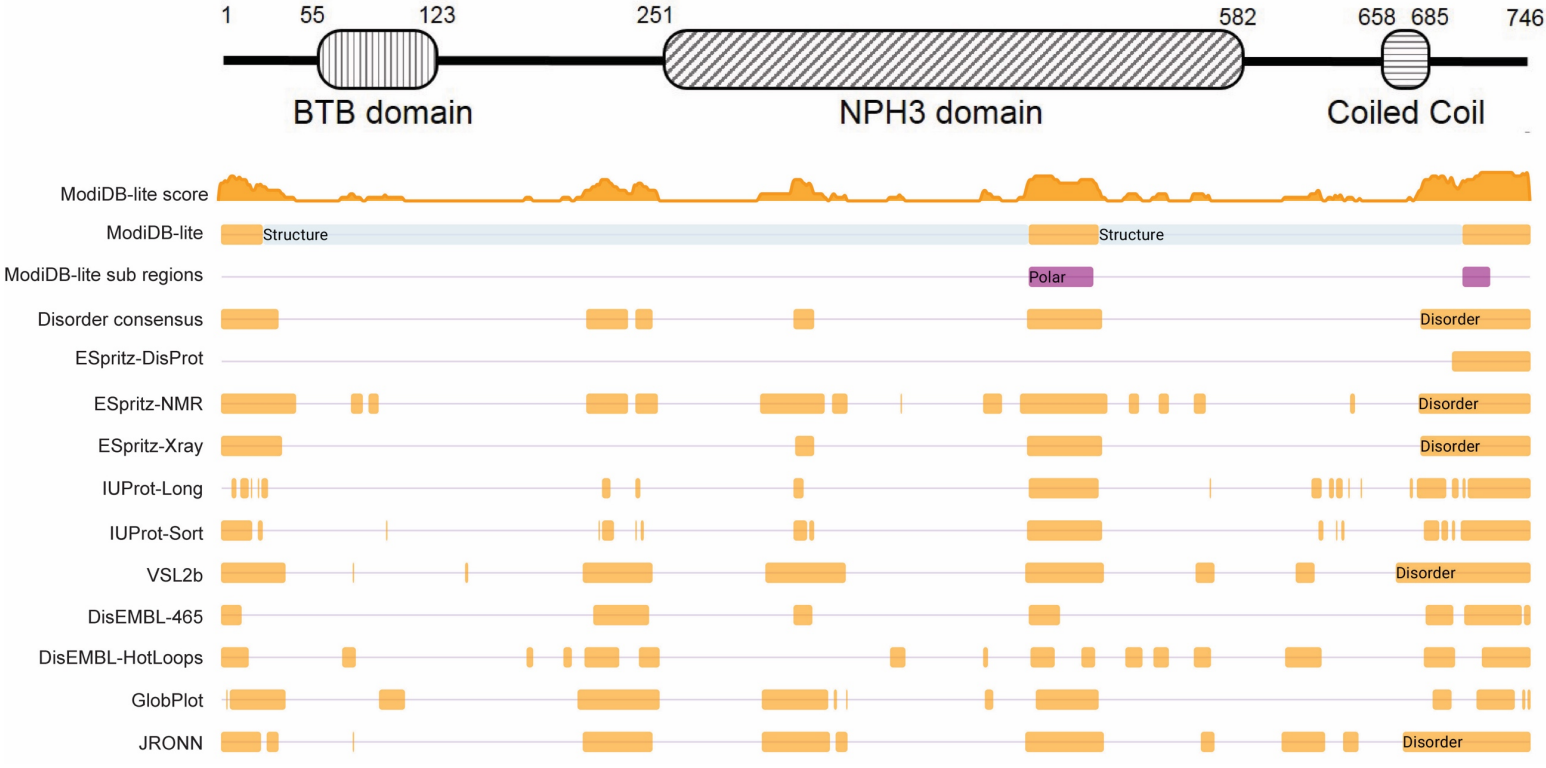

Fig. S1:

Domain structure of NPH3 and MobiDB plot (https://mobidb.org/) of intrinsically disordered regions in NPH3. BTB domain, broad-complex, tramtrack, bric a brac domain. 


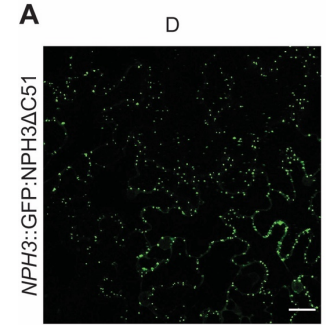

B
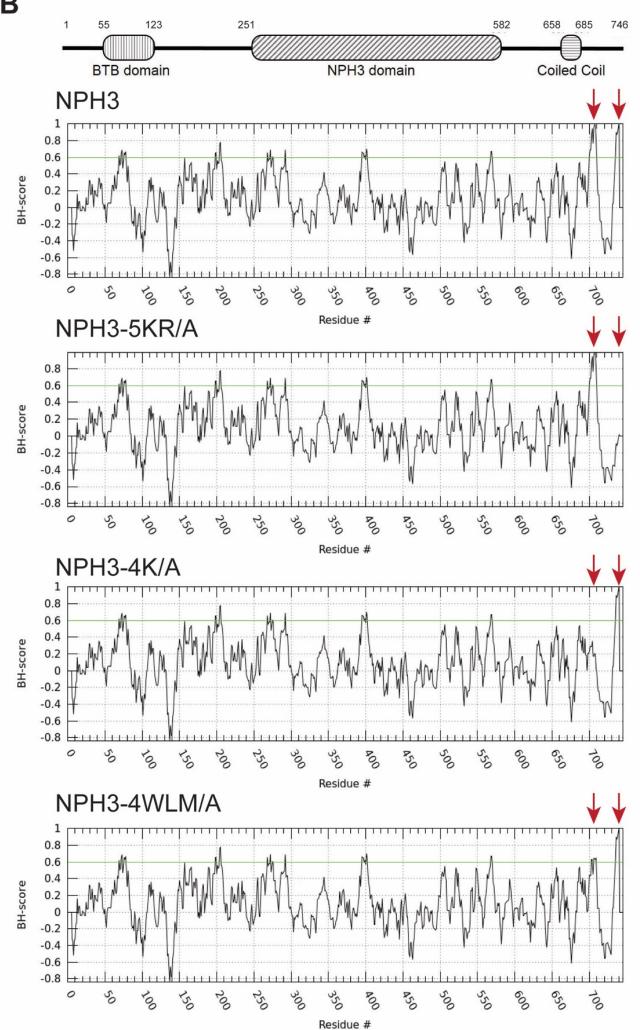

D

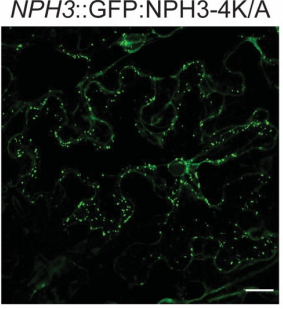

$\mathrm{E}$

35S::GFP:NPH3 $\triangle \mathrm{C} 51$

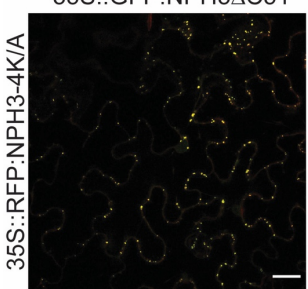

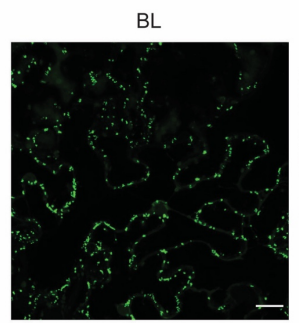

NPH3.:GFP.NPH3-4WLMIA

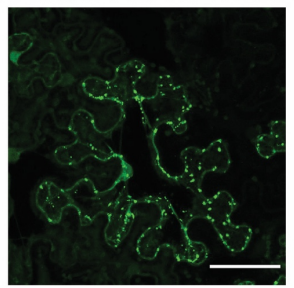

35S::GFP:NPH3 3 C 51

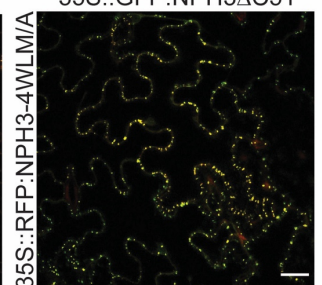

D

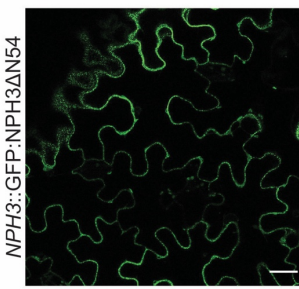

C
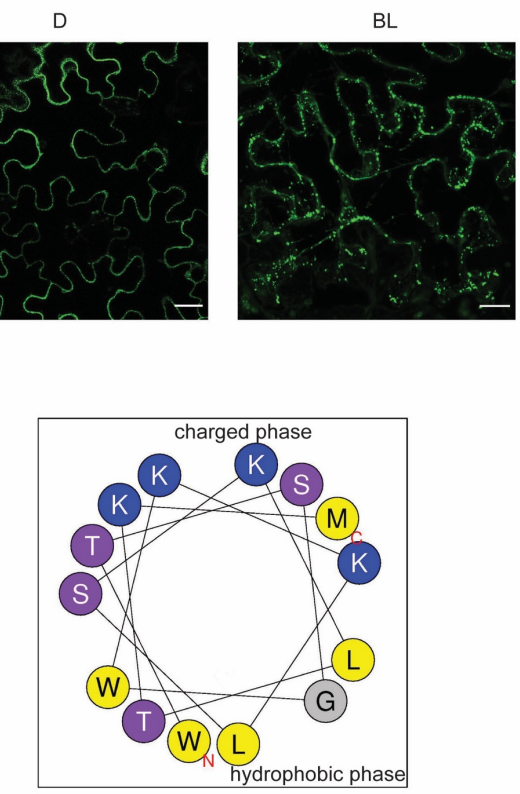

$\mathrm{NPH} 3$

hydrophobicity $\mathrm{H} \quad 0.401$ hydrophobic moment $\mu \mathrm{H} \quad 0.580$ net charge

\section{NPH3-4K/A}

hydrophobicity $\mathrm{H} \quad 0.772$

hydrophobic moment $\mu \mathrm{H} \quad 0.316$

net charge

NPH3-4WLM/A

hydrophobicity $\mathrm{H}$

net charge

0.401

$+4$

35S::RFP

NPH3-4K/A-S744A

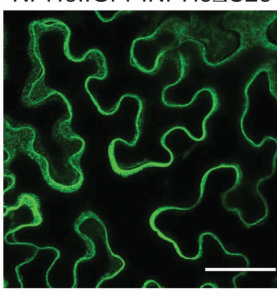

35S::GFP:NPH3-4WLM/A

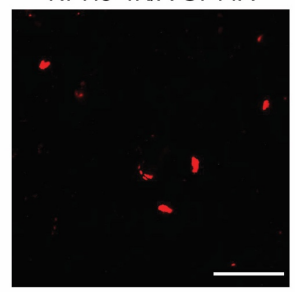

35S::GFP:

NPH3-4K/A-S744A
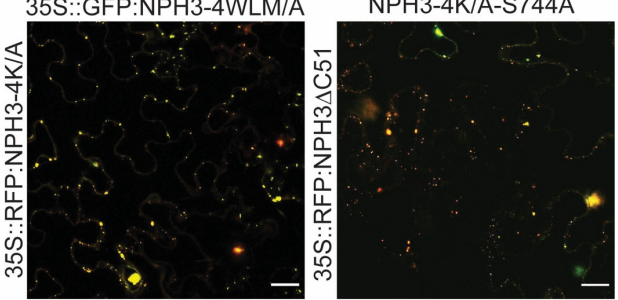

1009 Fig. S2:

(A), (D), (E) Representative confocal microscopy images of leaf epidermal cells from transiently transformed $N$. benthamiana. (A) The plants were either kept in darkness (D) or treated with BL (approx.11 min by means of the GFP-laser, Z-stack projections are shown). (D, E) The plants were adapted to darkness (D: Z-stack projections). Scale bars, $25 \mu \mathrm{m}$. (B) BH score profiles (window size 11) of NPH3 and mutant variants. Putative BH-domains are indicated by red arrows. 
1016 (C) Helical wheel projection showing amphipathy of the predicted helix (residues 700-713) within the $\mathrm{C}$-terminal domain of $\mathrm{NPH} 3$. Overall helix hydrophobicity $(\mathrm{H})$ and the hydrophobic moment $(\mu \mathrm{H})$ are given for $\mathrm{NPH} 3$ and mutant variants. 

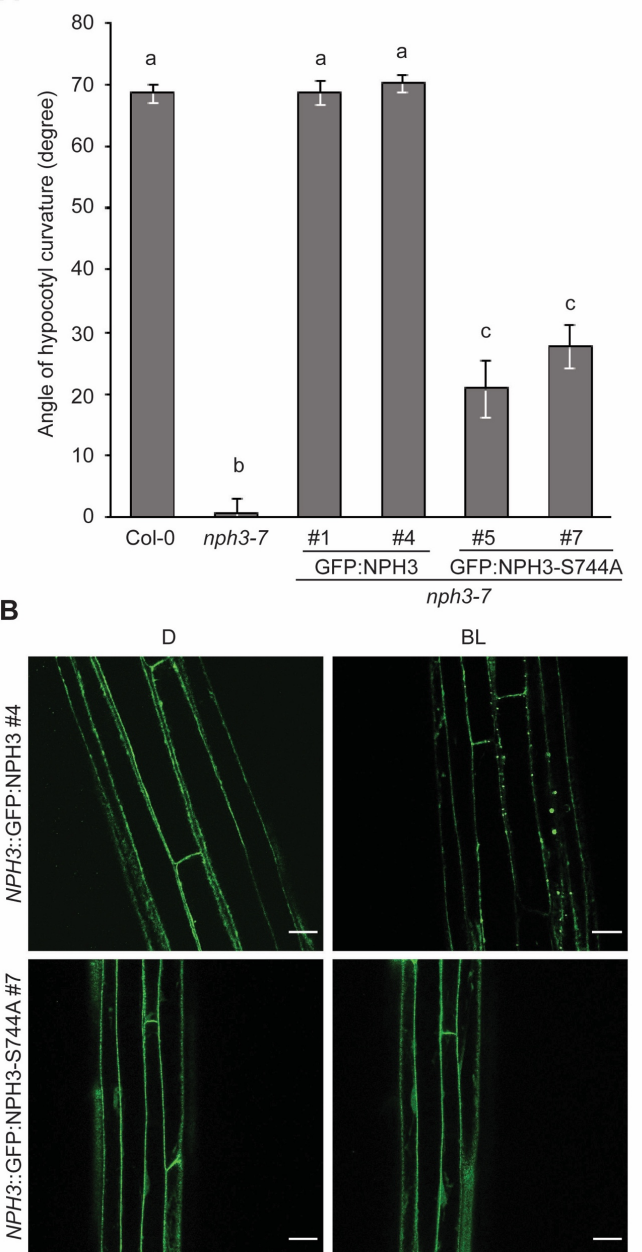

$\mathrm{BL}$
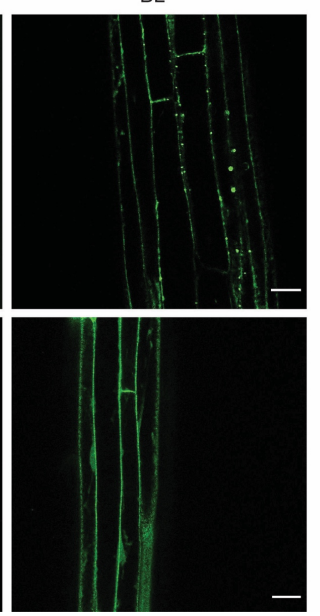

C

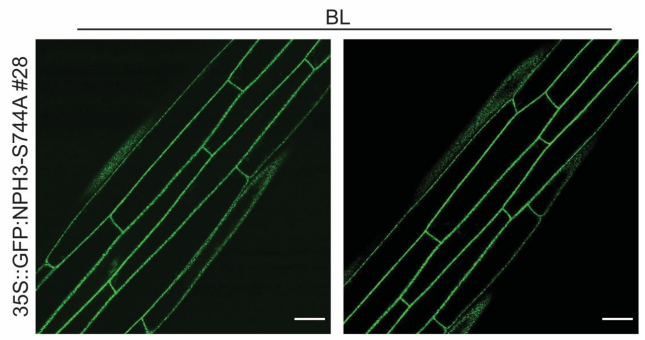

35S::14-3-3:GFP +

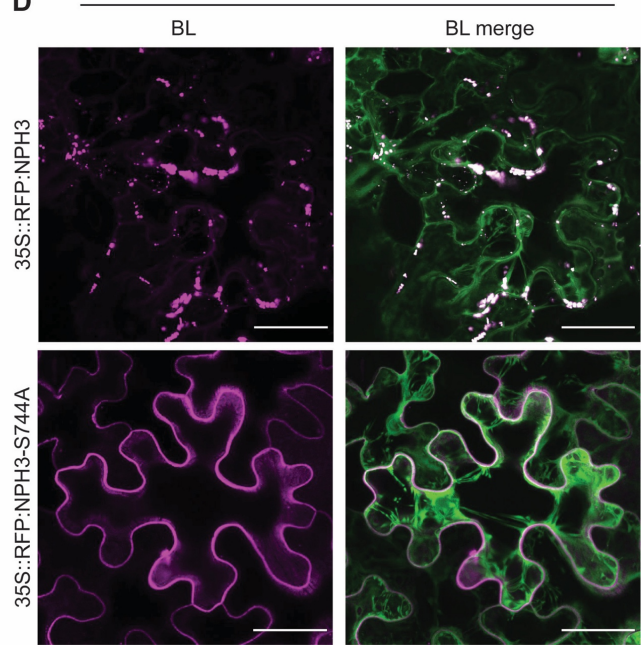

Fig. S3:

(A) Quantification of the hypocotyl phototropism response (mean \pm SEM) in 3-days old etiolated seedlings exposed for $12 \mathrm{~h}$ to unilateral blue light $\left(1 \mu \mathrm{mol} \mathrm{m}^{-2} \mathrm{sec}^{-1}\right)(n>30$ seedlings per experiment, one representative experiment of two replicates is shown). Expression of transgenes in $n p h 3-7$ was driven by the NPH3 promoter. Student's t-test, different letters mark statistically significant differences $(P<0.05)$, same letters mark statistically nonsignificant differences.

(B, C, D) Representative confocal microscopy images of hypocotyl cells from transgenic etiolated Arabidopsis $n p h 3-7$ seedlings (B, C) or of leaf epidermal cells from transiently transformed $N$. benthamiana (here, Z-stack projections are shown) (D). The plants were either kept in darkness (D) or treated with blue light (BL) (nph3-7: $1 \mu \mathrm{mol} \mathrm{m} \mathrm{m}^{-2} \mathrm{sec}^{-1}$ and $N$. benthamiana: $\left.10 \mu \mathrm{mol} \mathrm{m}{ }^{-2} \mathrm{sec}^{-1}\right)$ for $40 \mathrm{~min}$. Expression of transgenes was driven by the NPH3 promoter (B) or the 35S promoter (C, D). Scale bars, $25 \mu \mathrm{m}$. 


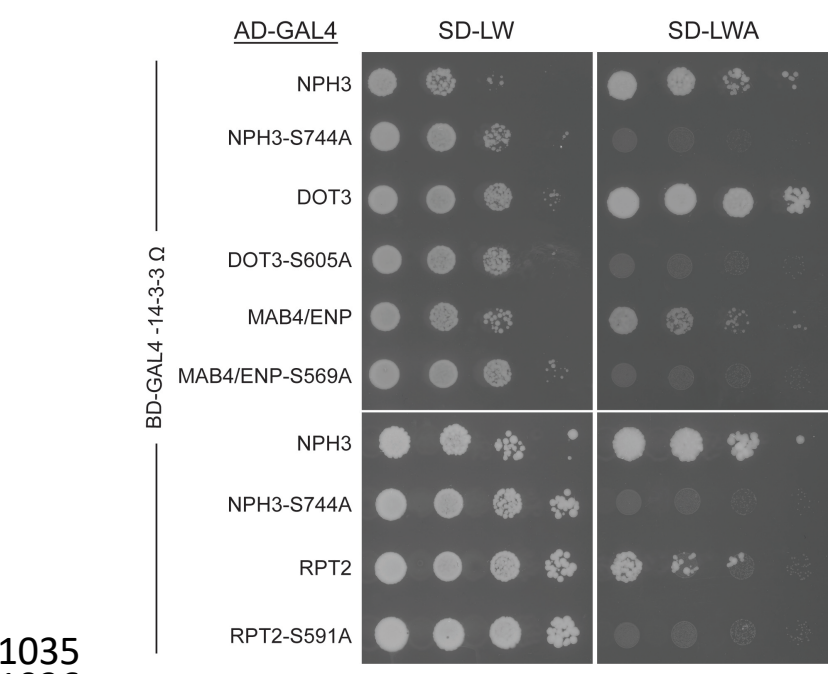

Fig. S4:

Yeast two-hybrid interaction analysis of the Arabidopsis 14-3-3 isoform omega with various NRL wild type and mutant variants (exchange of the antepenultimate residue (serine), respectively). Yeast growth was recorded after 3 days (upper panel) or 5 days (lower panel). 
Analysis of 14-3-3 epsilon-GFP immunoprecipitates via mass spectrometry (MS) based on two biological replicates. This table lists only known 14-3-3 clients in addition to NPH3.

\begin{tabular}{|c|c|c|c|c|c|c|c|c|c|c|c|c|}
\hline \multirow[b]{2}{*}{ AGI code } & \multirow[b]{2}{*}{ gene name } & \multirow[b]{2}{*}{ description } & \multirow{2}{*}{$\begin{array}{l}\text { Mol. weight } \\
(\mathrm{kDa})\end{array}$} & \multicolumn{2}{|c|}{ peptides R1 } & \multicolumn{2}{|c|}{ Sequence coverage R1 (\%) } & \multicolumn{2}{|c|}{ Intensity R1 } & \multicolumn{2}{|c|}{ Normalized intensity R1 } & \multirow{2}{*}{$\begin{array}{c}\text { Intensity (BL/dark) } \\
\text { R1 }\end{array}$} \\
\hline & & & & dark & BL & dark & BL & dark & BL & dark & BL & \\
\hline AT1G22300 & GRF10 & 14-3-3-like protein GF14 epsilon & 28,9 & 32 & 30 & 79,5 & 79,5 & $3,2308 E+11$ & $3,7406 E+11$ & $3 \mathrm{E}+11$ & $3 E+11$ & 1 \\
\hline AT1G35580 & CINV1 & Alkaline/neutral invertase CINV1 & 62,834 & 35 & 32 & 64,2 & 64,2 & $3,4953 \mathrm{E}+10$ & $2,9811 E+10$ & $3,245 \mathrm{E}+10$ & $2,391 \mathrm{E}+10$ & 0,735 \\
\hline AT2G18960 & AHA1 & ATPase 1, plasma membrane-type & 104,22 & 31 & 36 & 36,4 & 43,4 & 2970100000 & 4414600000 & 2760000000 & 3540000000 & 1,28 \\
\hline AT4G30190 & AHA2 & ATPase 2, plasma membrane-type & 104,4 & 33 & 37 & 39,9 & 42,6 & 401660000 & 380890000 & 372900000 & 305450000 & 0,819 \\
\hline AT5G64330 & $\mathrm{NPH} 3$ & Non-phototropic hypocotyl 3 & 81,872 & 5 & 22 & 7,4 & 39 & 53537000 & 1145400000 & 49700000 & 919000000 & 18,5 \\
\hline AT1G09570 & PhyA & Phytochrome A & 125,02 & 1 & 1 & 0,9 & 1,2 & 4661700 & 5509900 & 4328000 & 4419000 & 1,02 \\
\hline AT5G11110 & SPS1 & Sucrose-phosphate synthase 1 & 117,32 & 13 & 18 & 15,6 & 22,7 & 246270000 & 519340000 & 228700000 & 416500000 & 1,819 \\
\hline AT5G03280 & EIN2 & Ethylene-insensitive protein 2 & 140,95 & 6 & 9 & 6,6 & 9 & 81033000 & 136130000 & 75240000 & 109200000 & 1,45 \\
\hline \multirow[t]{2}{*}{ AT3G45780 } & Phot1 & Phototropin-1 & 111,69 & 10 & 14 & 8,8 & 14,7 & 169850000 & 316360000 & 157700000 & 253700000 & 1,61 \\
\hline & & & & \multicolumn{2}{|c|}{ peptides R2 } & \multicolumn{2}{|c|}{ Sequence coverage R2 (\%) } & \multicolumn{2}{|c|}{ Intensity R2 } & \multicolumn{2}{|c|}{ Normalized intensity R2 } & \\
\hline AGI code & gene name & description & (kDa) & dark & BL & dark & BL & dark & BL & dark & BL & R2 \\
\hline AT1G22300 & GRF10 & 14-3-3-like protein GF14 epsilon & 28,9 & 33 & 33 & 76,4 & 76,4 & $3,3401 E+11$ & $3,9971 E+11$ & $3 E+11$ & $3 E+11$ & 1 \\
\hline AT1G35580 & CINV1 & Alkaline/neutral invertase CINV1 & 62,834 & 31 & 30 & 61,2 & 57 & $2,879 \mathrm{E}+10$ & $4,4008 E+10$ & $2,586 \mathrm{E}+10$ & $3,303 \mathrm{E}+10$ & 1,275 \\
\hline AT2G18960 & AHA1 & ATPase 1, plasma membrane-type & 104,22 & 33 & 30 & 41,5 & 36,1 & 276600000 & 295590000 & 248400000 & 221900000 & 0,895 \\
\hline AT4G30190 & AHA2 & ATPase 2, plasma membrane-type & 104,4 & 34 & 31 & 38,9 & 36 & 3321800000 & 3478600000 & 2983000000 & 2611000000 & 0,87 \\
\hline AT5G64330 & NPH3 & Non-phototropic hypocotyl 3 & 81,872 & 1 & 14 & 1,5 & 24,4 & 0 & 502550000 & $0(1)$ & 377200000 & 377 \\
\hline AT1G09570 & PhyA & Phytochrome A & 125,02 & 4 & 1 & 5,1 & 1,6 & 21558000 & 4285500 & 19360000 & 3216000 & 0,166 \\
\hline AT5G11110 & SPS1 & Sucrose-phosphate synthase 1 & 117,32 & 8 & 9 & 12,8 & 14,4 & 135570000 & 237580000 & 121800000 & 178300000 & 1,4615 \\
\hline AT5G03280 & EIN2 & Ethylene-insensitive protein 2 & 140,95 & 5 & 3 & 5,1 & 2,7 & 48328000 & 46407000 & 43410000 & 35010000 & 0,807 \\
\hline AT3G45780 & Phot1 & Phototropin-1 & 111,69 & 8 & 3 & 9 & 3,4 & 85979000 & 75314000 & 77220000 & 56530000 & 0,73 \\
\hline
\end{tabular}

\section{Video S1:}

Dynamic BL-induced changes in the subcellular localization of 35S::GFP:NPH3 in hypocotyl cells of stably transformed Arabidopsis nph3-7.

\section{Video S2:}

Subcellular localization of 35S::GFP:NPH3-S744A in hypocotyl cells of stably transformed Arabidopsis nph3-7 upon BL-irradiation.

\section{Video S3:}

Dynamic BL-induced changes in the subcellular localization of 35S::RFP:NPH3 transiently expressed in $N$. benthamiana leaves.

\section{Video S4:}

Subcellular localization of 35S::RFP:NPH3-S744A in transiently transformed $N$. benthamiana leaves upon BL-irradiation.

\section{Video S5:}

Subcellular localization of 35S::GFP:NPH3-4K/A in hypocotyl cells of stably transformed Arabidopsis nph3-7 upon BL-irradiation.

\section{Video S6:}

Subcellular localization of 35S::GFP:NPH3 $\triangle \mathrm{C} 51$ in hypocotyl cells of stably transformed Arabidopsis nph3-7 upon BL-irradiation.

\section{Video S7:}

Subcellular localization of 35S::GFP:NPH3 $\Delta$ C28 in hypocotyl cells of stably transformed Arabidopsis nph3-7 upon BL-irradiation.

\section{Video S8:}

Dynamic BL-induced changes in the subcellular localization of 35S::RFP:NPH3 $\Delta$ N54 transiently expressed in $N$. benthamiana leaves.

\section{Video S9:}

Dynamic BL-induced changes in the subcellular localization of 35S::GFP:NPH3 $\Delta$ N54 in hypocotyl cells of stably transformed Arabidopsis nph3-7. 


\section{Figures}

A

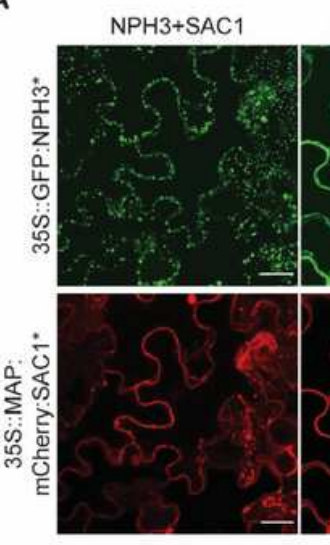

B

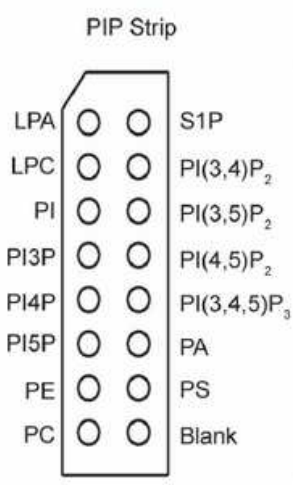
$\mathrm{NPH} 3+\mathrm{SAC} 1_{\text {DEAD }}$
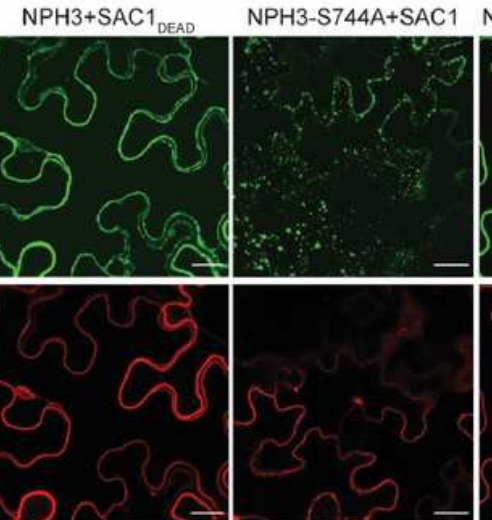

$\begin{array}{cc}\text { HA: } & \text { HA: } \\ \text { NPH3 } & \text { NPH3 } \triangle \mathrm{C} 5\end{array}$
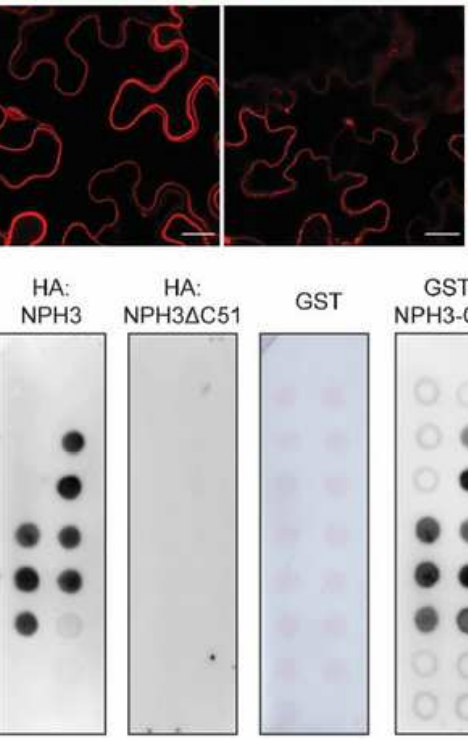

GST

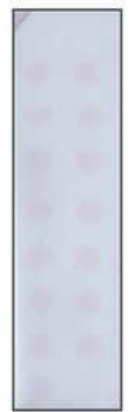

C
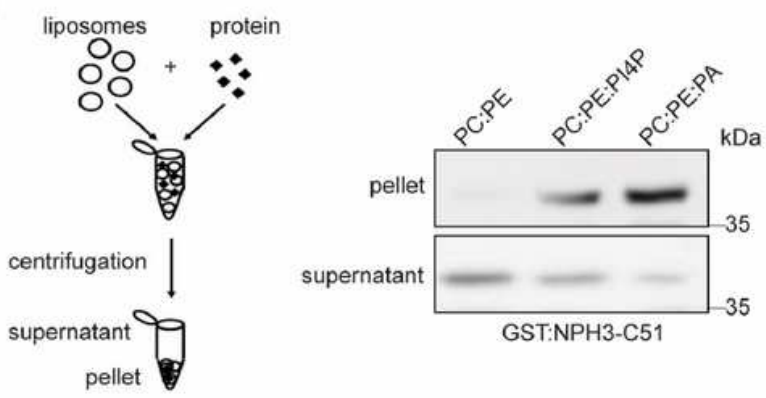

GST:NPH3-C51

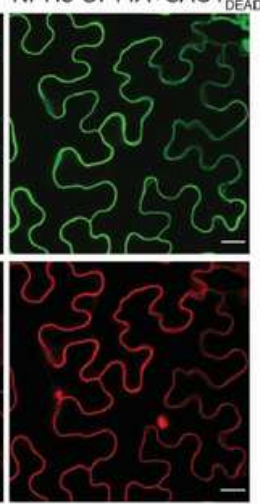

D $\quad \mathrm{NPH} 3 \triangle \mathrm{C} 51$

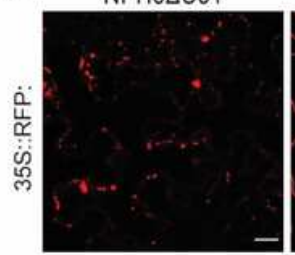

NPH3-S744D

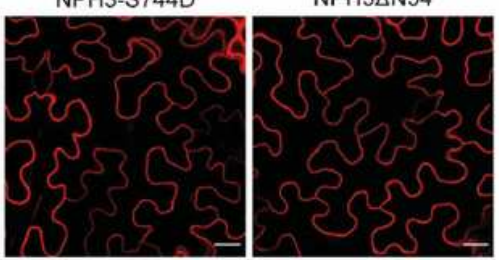

$\mathrm{E}$
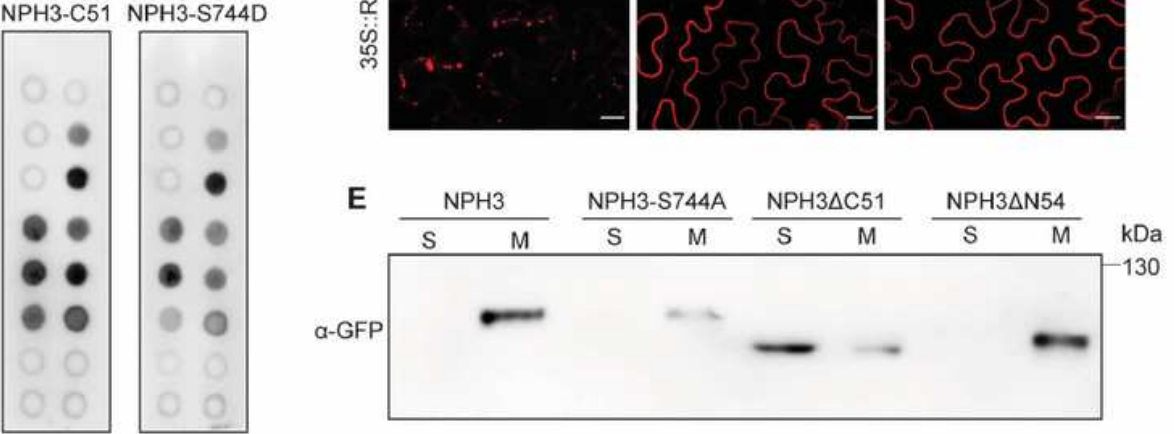

\section{Figure 1}

NPH3 binds to polyacidic phospholipids via its C-terminal domain. (A), (D) Representative confocal microscopy images of leaf epidermal cells from transiently transformed $\mathrm{N}$. benthamiana adapted to darkness (Z-stack projections of NPH3DC51 (D) as well as NPH3 variants (NPH3*) co-expressed with SAC1 variants $\left(\mathrm{SAC1}^{*}\right)(\mathrm{A})$ are shown). Scale bars, $25 \mu \mathrm{m}$. (B) Lipid overlay assay performed with either in vitro transcribed and translated HA:NPH3 and HA:NPH3DC51 or purified GST and GST:NPH3-C51 variants. Immunodetection was performed by using anti-HA or anti-GST antibodies, respectively. See main text for abbreviations. (C) Liposome binding assay using large unilamellar liposomes containing the neutral phospholipids PE and PC mixed with either the polyacidic PI4P or PA as specified. Anti-GST immunoblot of GST:NPH3-C51 is shown. (E) Representative immunoblots with anti-GFP after subcellular fractionation of protein extracts prepared from $\mathrm{N}$. benthamiana leaves transiently expressing 35S::GFP:NPH3 variants and adapted to darkness. Proteins in each fraction $(7.5 \mu \mathrm{g})$ were separated on $7.5 \%$ SDS-PAGE gels. Note that the total amount of soluble proteins (S) is approximately 15 times higher as compared to the total amount of microsomal proteins (M) after 100,000 g centrifugation. 

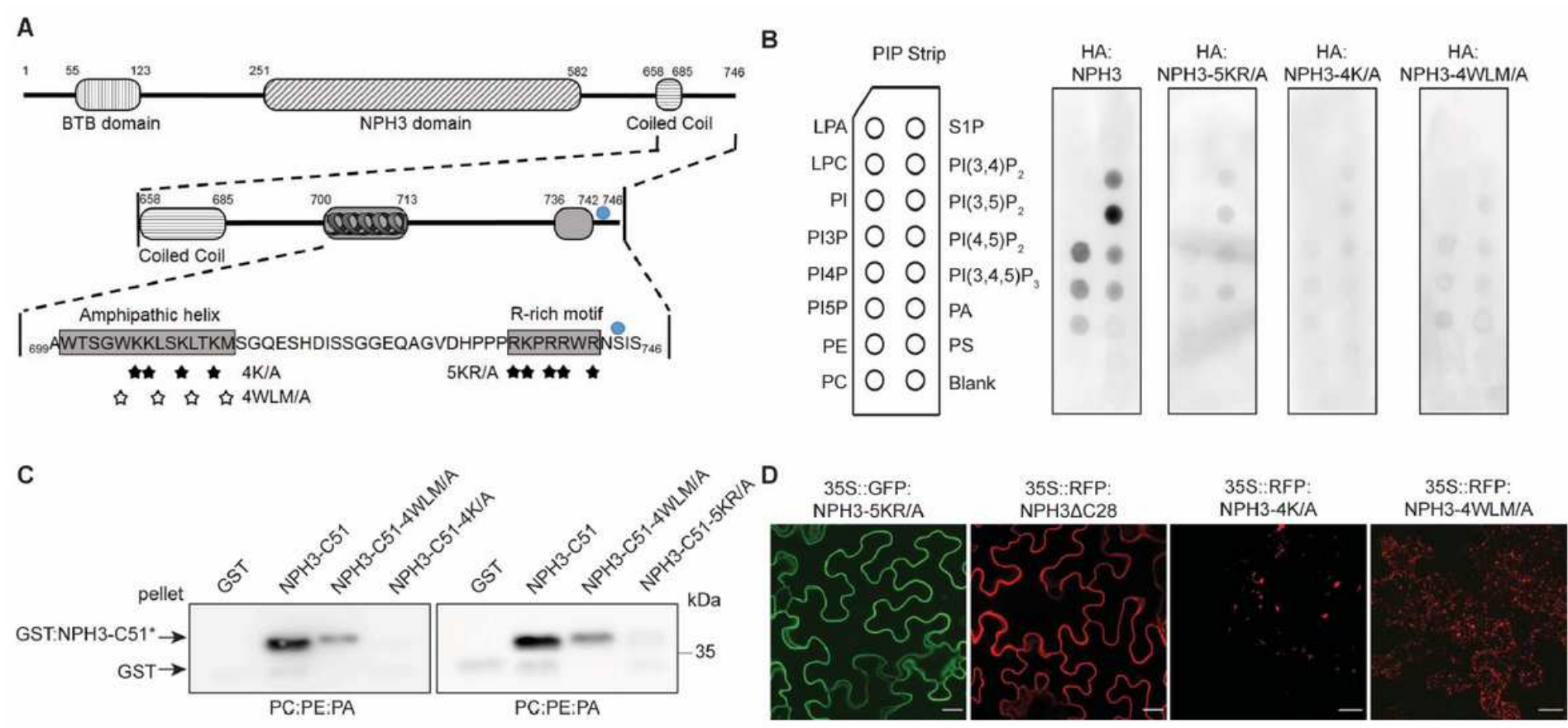

Figure 2

An amphipathic helix within the $\mathrm{C}$-terminal domain is required for NPH3 phospholipid binding, membrane association and plasma membrane localization. (A) Domain structure and primary sequence of NPH3 showing the two putative BH domains (amphipathic helix and R-rich motif) within the C-terminal region. Stars depict residues of either the R-rich motif or the amphipathic helix substituted by alanine $(A)$ in the NPH3 variants, blue circle depicts the 14-3-3 binding site (see Fig. 3). (B) Lipid overlay assay performed with purified GST:NPH3-C51 variants (C51*). (C) Liposome binding assay using large unilamellar liposomes containing the neutral PE and PC mixed with the polyacidic PA. Anti-GST immunoblot of GST:NPH3-C51 variants is shown. (D) Representative confocal microscopy images of leaf epidermal cells from transiently transformed N. benthamiana adapted to darkness (Z-stack projections of NPH3-4K/A and NPH3-4WLM/A are shown). Scale bars, $25 \mu \mathrm{m}$. 
A

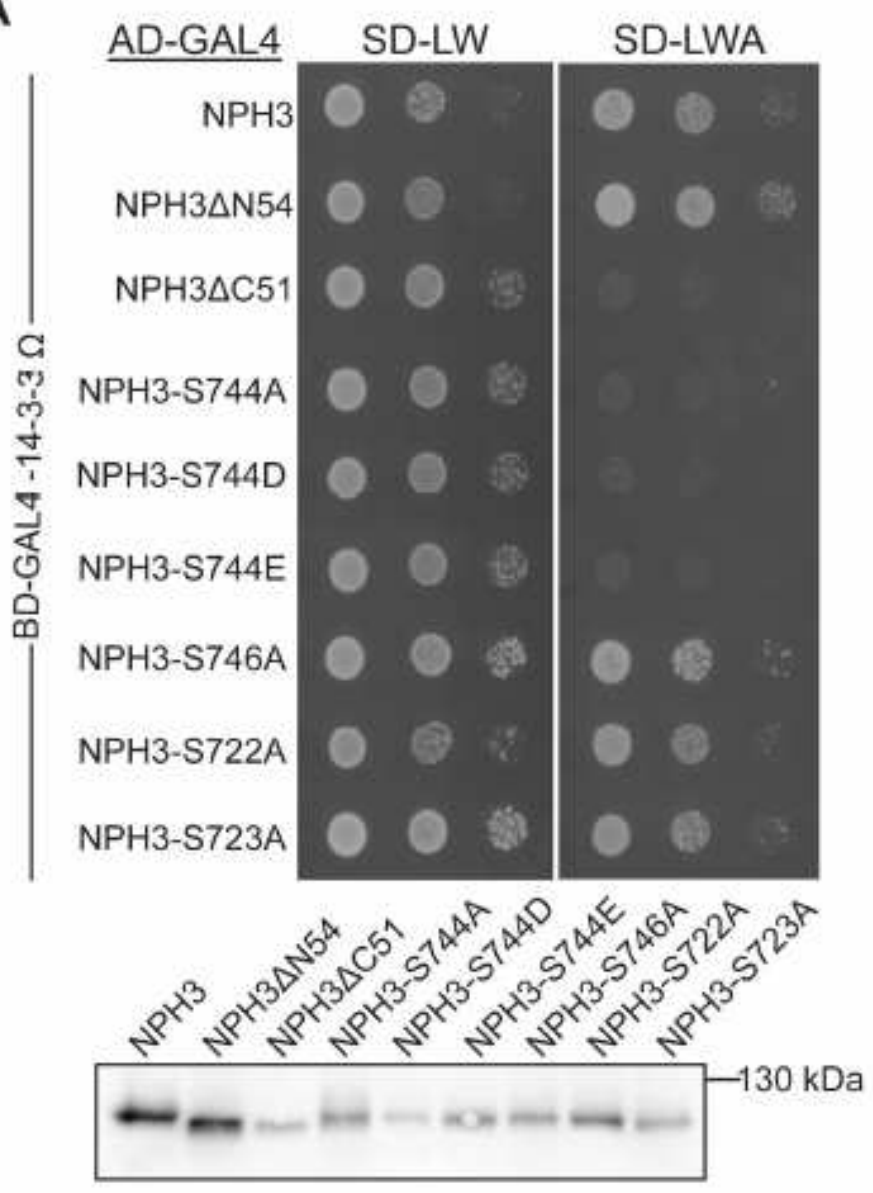

B

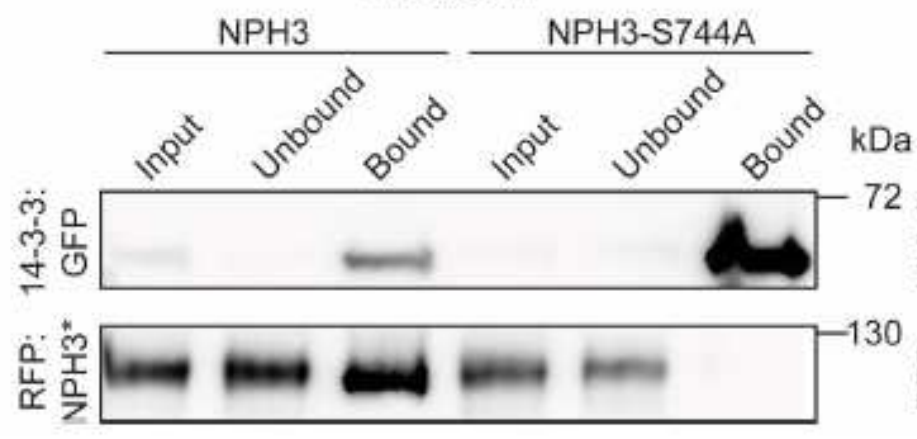

C

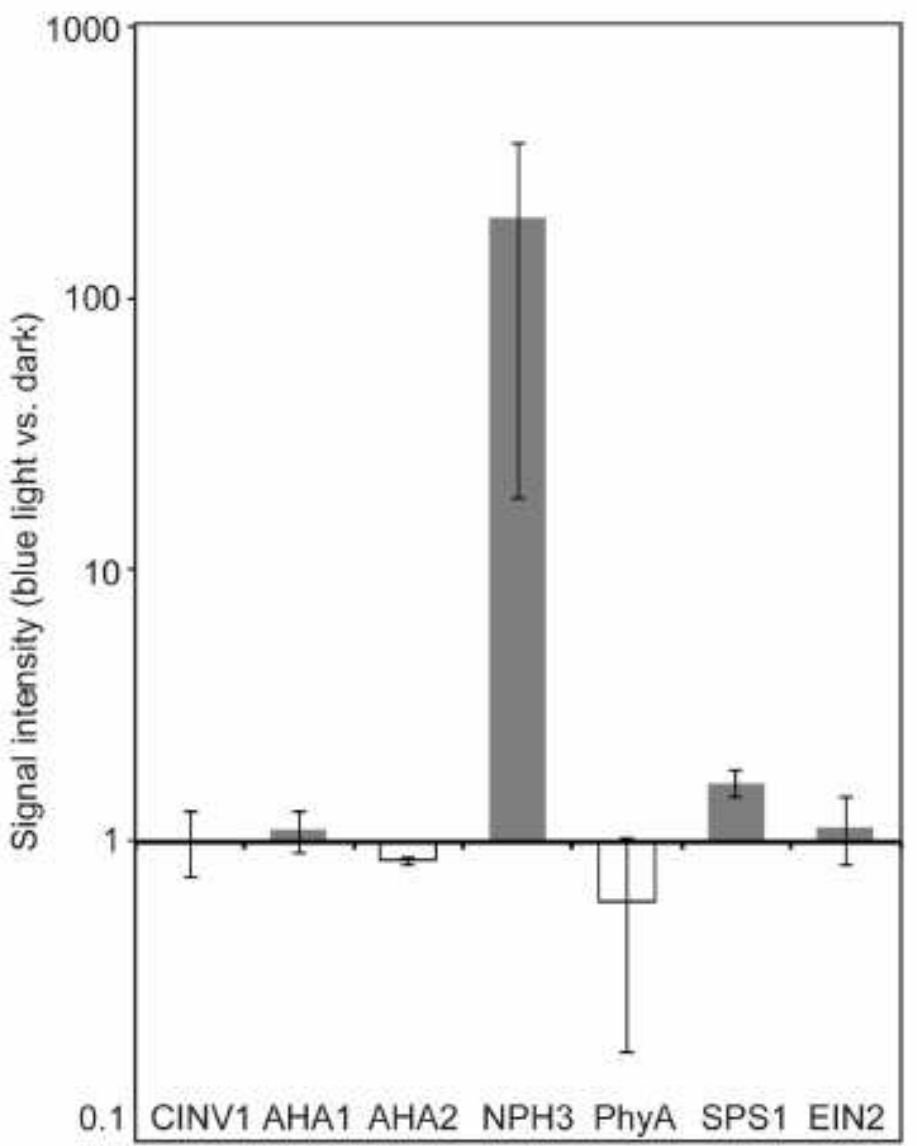

IP: $\alpha-G F P$

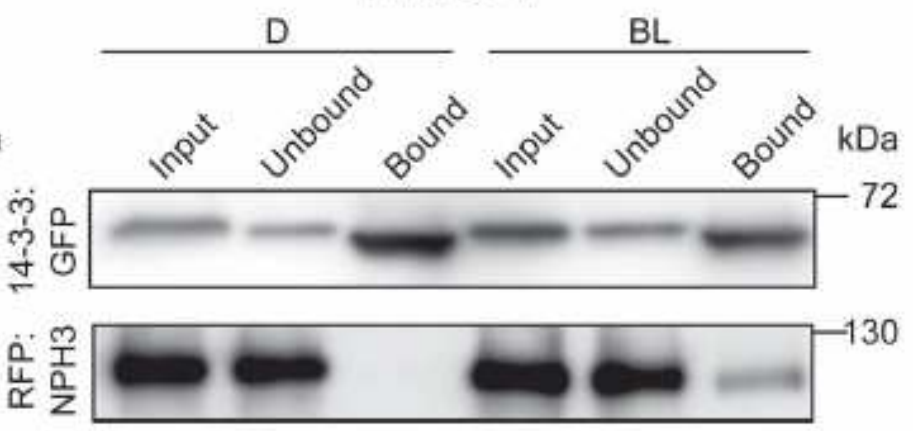

\section{Figure 3}

Interaction of NPH3 and 14-3-3 proteins is triggered by blue light irradiation and abolished by mutation of the antepenultimate NPH3 residue. (A) Yeast two-hybrid interaction analysis of the Arabidopsis 14-3-3 isoform omega with NPH3 wild type and mutant variants (upper panel). Expression of the diverse NPH3 fusion proteins in yeast was confirmed by anti-HA-immunodetection (lower panel). AD, activating domain; $B D$, binding domain. $(B, D)$ In vivo interaction of mCherry:NPH3 variants and 14-3-3 omega:mEGFP in transiently transformed $\mathrm{N}$. benthamiana leaves. Expression of transgenes was driven by the $35 \mathrm{~S}$ promoter. Freshly transformed tobacco plants were either kept under constant light for $42 \mathrm{~h}$ (B) or kept under constant light for $24 \mathrm{~h}$ and subsequently transferred to darkness for $17 \mathrm{~h}$ with (BL) or without (D) 
blue light treatment ( $5 \mu \mathrm{mol} \mathrm{m}-2 \mathrm{sec}-1)$ for the last 40 minutes (D). The crude extract was immunoprecipitated using GFP beads and separated on 11\% SDS-PAGE gels, followed by immunoblotting with anti-GFP and anti-RFP antibodies, respectively. (C) Arabidopsis 14-3-3 epsilon interactors were identified by mass spectrometry analysis of anti-GFP immunoprecipitations (two biological replicates) from etiolated seedlings expressing 14-3-3 epsilon:GFP either maintained in darkness or irradiated with blue light $(1 \mu \mathrm{mol} \mathrm{m}-2 \mathrm{sec}-1)$ for $30 \mathrm{~min}$. Protein intensities of 14-3-3 client proteins were normalized to relative abundance of the bait protein (Table S1). Fold changes in relative abundance (mean $\pm S D$, logarithmic scale) of blue light treatment versus darkness are given. AHA1, AHA2, Arabidopsis H+-ATPase; CINV1, cytosolic invertase 1; EIN2, ethylene insensitive 2; PhyA, phytochrome A; SPS1, sucrose phosphate synthase 1. 
A

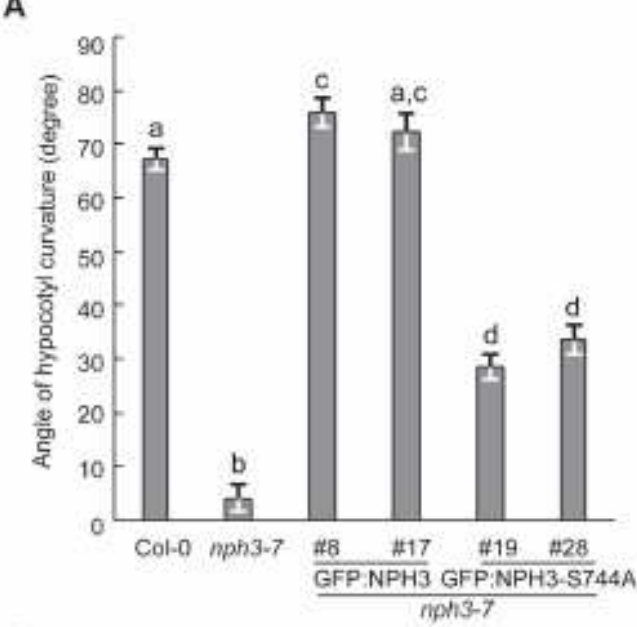

C

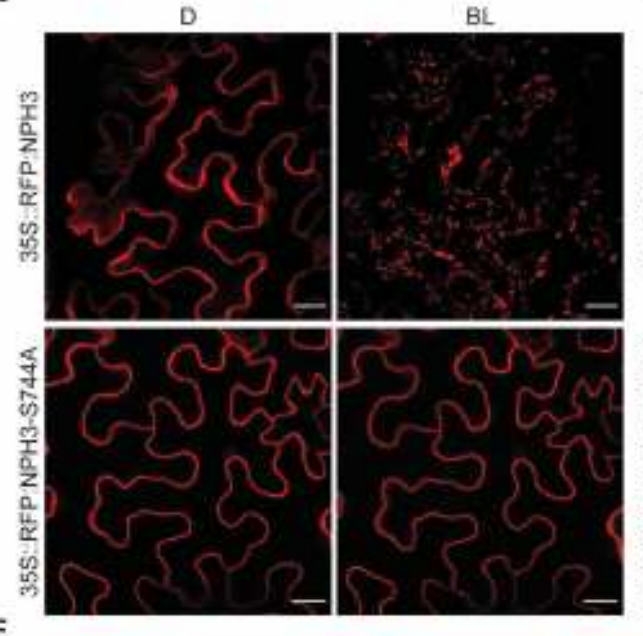

E

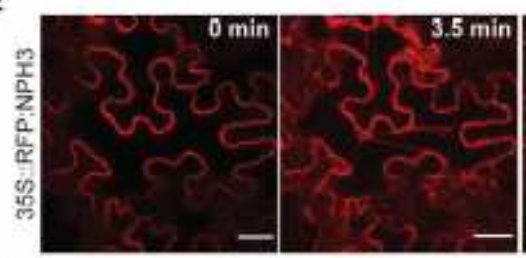

F

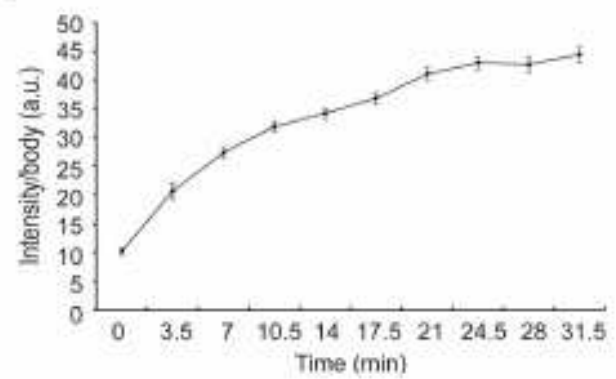

B

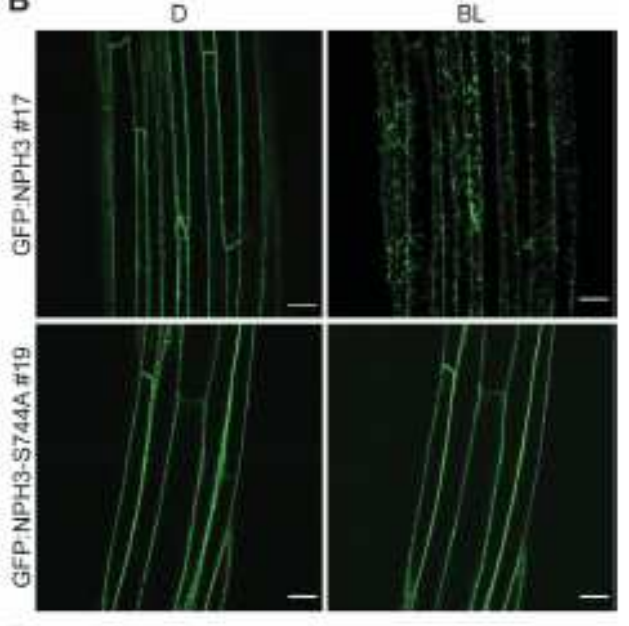

$B L$
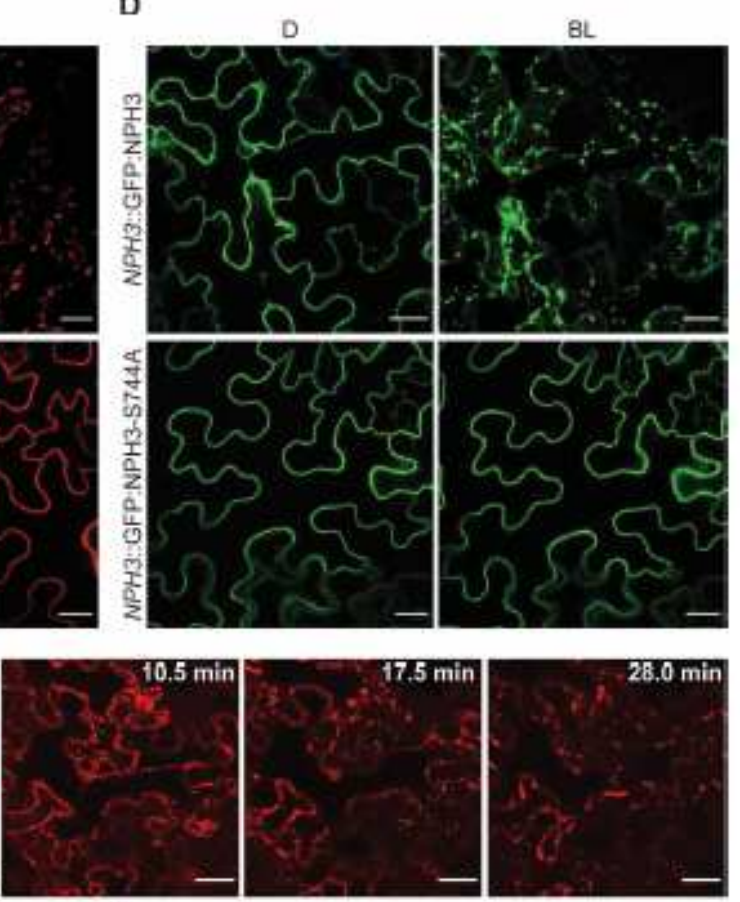

G

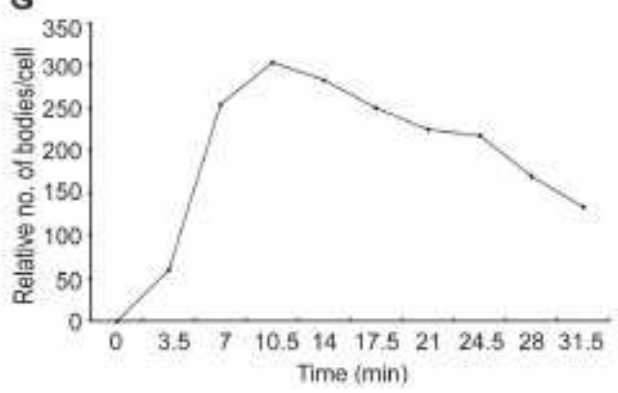

\section{Figure 4}

14-3-3 binding is required for proper NPH3 function in phototropic hypocotyl bending and its lighttriggered detachment from the plasma membrane. (A) Quantification of the hypocotyl phototropism response (mean \pm SEM) in 3-days old etiolated seedlings exposed for $12 \mathrm{~h}$ to unilateral blue light ( $1 \mu \mathrm{mol}$ m-2 sec-1) ( $n>30$ seedlings per experiment, one representative experiment of two replicates is shown). Expression of transgenes in nph3-7 was driven by the 35S promoter. Student's t-test, different letters mark 
statistically significant differences $(P<0.05)$, same letters mark statistically non-significant differences. (B, C, D) Representative confocal microscopy images of hypocotyl cells from transgenic etiolated Arabidopsis nph3-7 seedlings (B) or of leaf epidermal cells from transiently transformed N. benthamiana (Z-stack projections of BL-treated NPH3 are shown) (C, D). The plants were either kept in darkness (D) or treated with blue light (BL) (N. benthamiana: approx. $11 \mathrm{~min}$ and nph3-7: approx. 6 min by means of the GFP-laser). Expression of transgenes was driven by the 35 S promoter $(\mathrm{B}, \mathrm{C})$ or the native NPH3 promoter (D). Scale bars, $25 \mu \mathrm{m}$. (E, F, G) Single-cell time-lapse imaging of RFP: NPH3 condensation induced by GFP-laser treatment. The image of time point 0 image was taken in the absence of the GFP-laser. $Z$ stack projections from selected time points (E), fluorescence intensity per body (mean $\pm S E M)(F)$ and number of bodies (G) are shown. Scale bars, $25 \mu \mathrm{m}$.
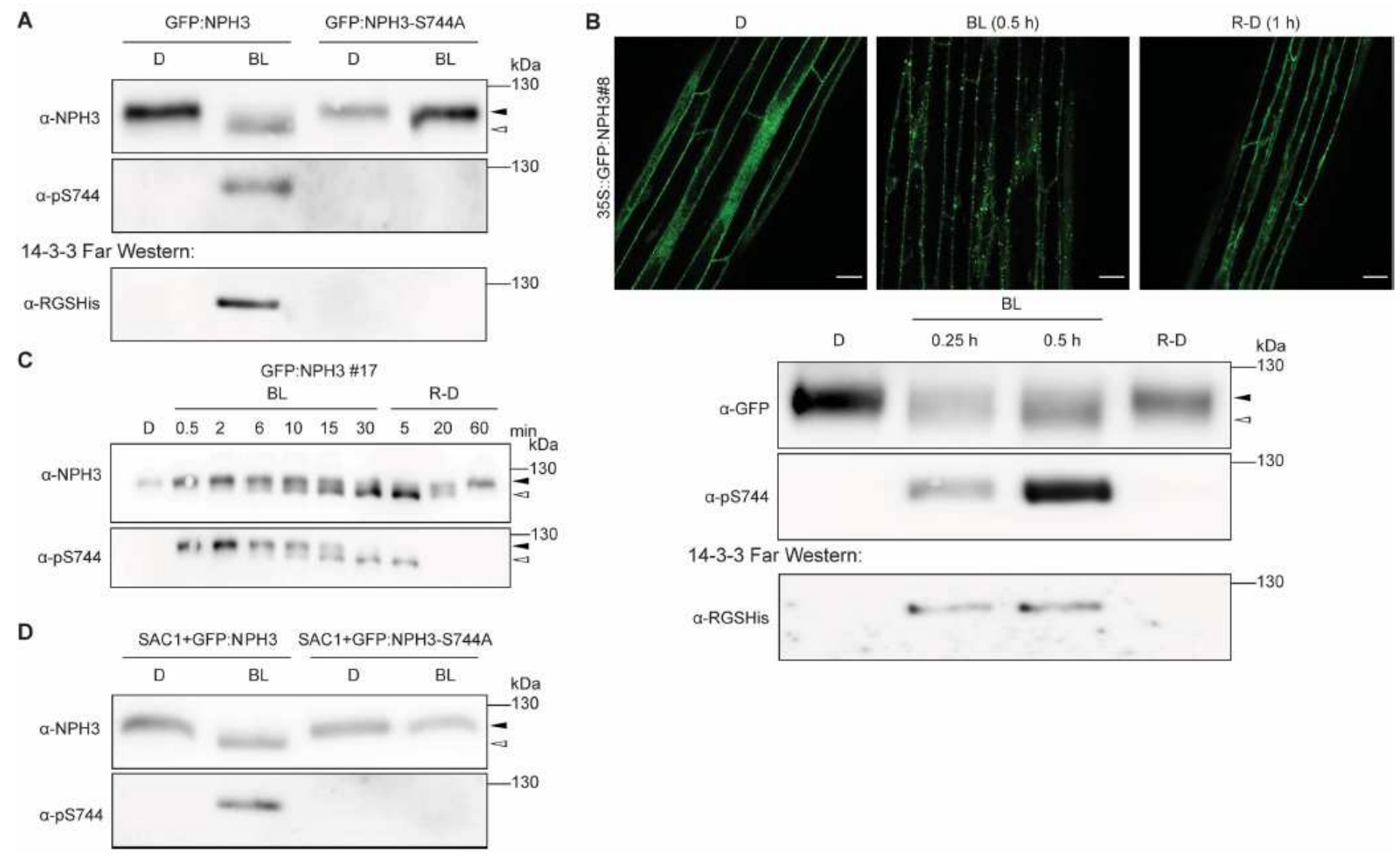

\section{Figure 5}

The phosphorylation status of the NPH3 14-3-3 binding site is dynamically modulated by the light regime. $(A, B, C)$ Immunoblot analysis of total protein extracts (C) or anti-GFP immunoprecipitates and 14-3-3 FarWestern (A, B) from Arabidopsis nph3-7 ectopically expressing GFP:NPH3 or GFP:NPH3-S744A. 3-days old etiolated seedlings were treated with cycloheximide $(100 \mu \mathrm{M})$ for $1 \mathrm{~h}(\mathrm{~B})$ and either maintained in darkness (D), treated with blue light (BL) $(1 \mu \mathrm{mol} \mathrm{m}-2$ sec-1) for the indicated time (A: $30 \mathrm{~min})$, or retransferred to darkness $(1 \mathrm{~h})$ after 30 min of irradiation (R-D). Proteins were separated on $7.5 \%$ SDS-PAGE gels. The upper panel in (B) shows representative confocal microscopy images of hypocotyl cells from transgenic etiolated Arabidopsis seedlings under the specified conditions. Scale bars, $25 \mu \mathrm{m}$. (D) 
Immunoblot analysis of transiently transformed N. benthamiana leaves co-expressing SAC1:RFP with either GFP:NPH3 or GFP:NPH3-S744A and adapted to darkness (see Fig. 1A). Expression of transgenes was driven by the $35 \mathrm{~S}$ promoter. Total protein extracts were separated on 7.5\% SDS-PAGE gels. The closed and open arrowheads indicate the positions of 'generally' phosphorylated and dephosphorylated $\mathrm{NPH} 3$ proteins, respectively.
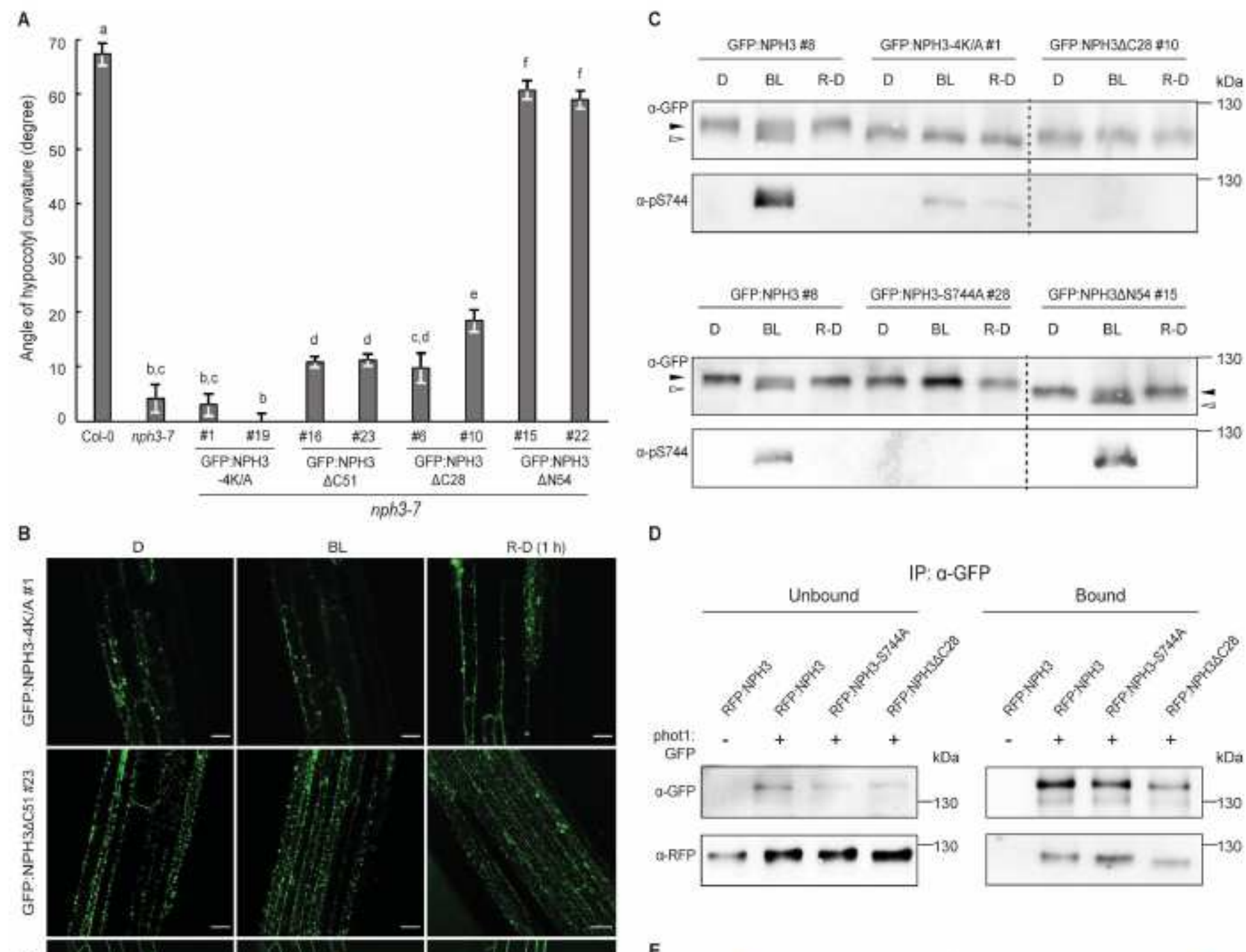

D
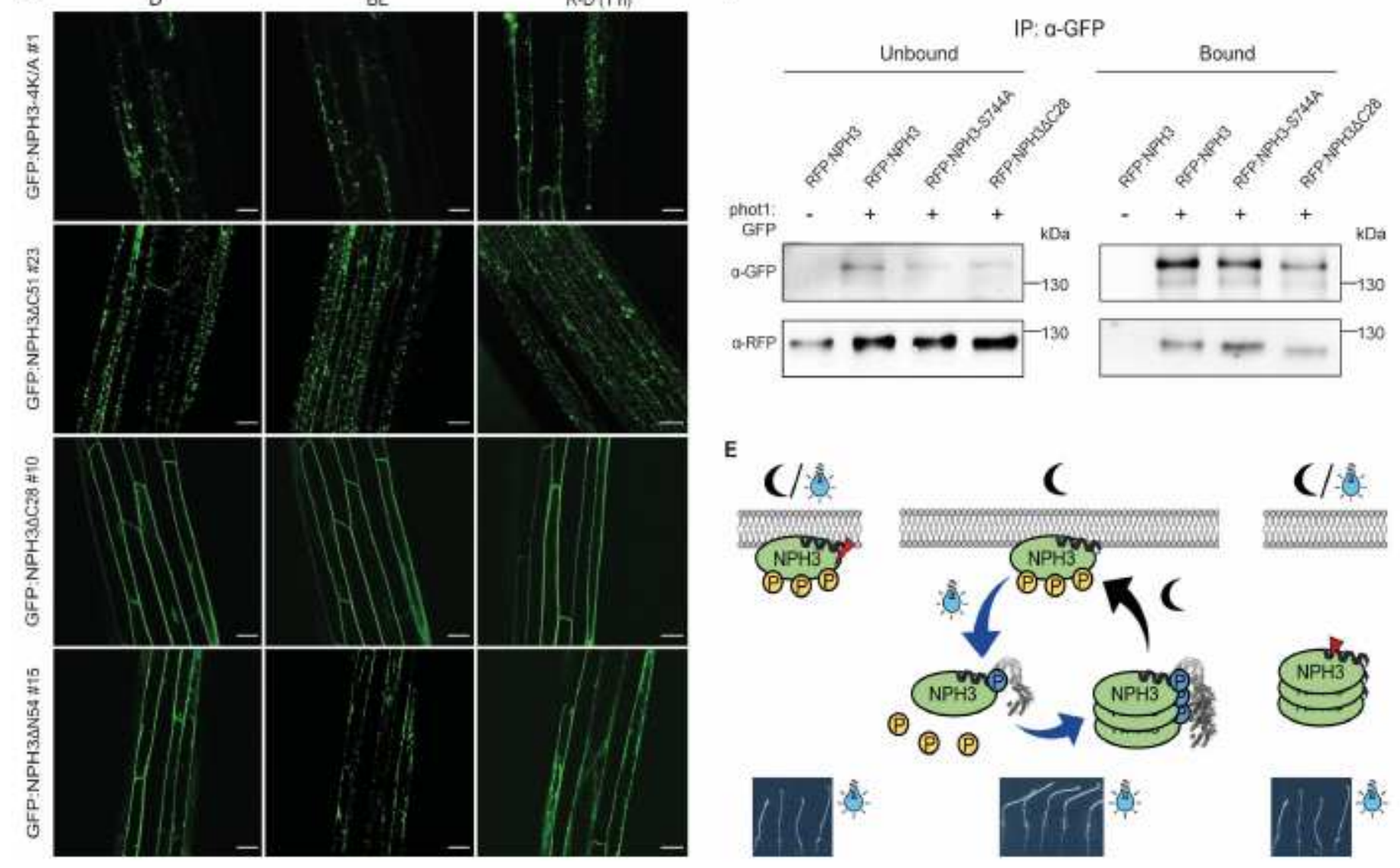

Figure 6 
Functional relevance of the subcellular localization of NPH3. (A) Quantification of the hypocotyl phototropism response (mean \pm SEM) in 3-days old etiolated seedlings exposed for $12 \mathrm{~h}$ to unilateral blue light $(1 \mu \mathrm{mol} m-2$ sec-1) $(n>30$ seedlings per experiment, one representative experiment of two replicates is shown). Expression of wild-type and mutant variants of GFP:NPH3 in nph3-7 was driven by the 35S promoter. Student's t-test, different letters mark statistically significant differences $(P<0.05)$, same letters mark statistically non-significant differences. (B) Representative confocal microscopy images of hypocotyl cells from transgenic Arabidopsis nph3-7 seedlings ectopically expressing mutant variants of GFP:NPH3. 3-days old etiolated seedlings were either maintained in darkness (D), treated with blue light (BL) (approx. 11 min by means of the GFP-laser) or re-transferred to darkness (1 h) (R-D) after 30 min of irradiation ( $1 \mu \mathrm{mol} \mathrm{m}-2 \mathrm{sec}-1)$. Scale bars, $25 \mu \mathrm{m}$. (C) Immunoblot analysis of etiolated Arabidopsis nph37 seedlings ectopically expressing mutant variants of GFP:NPH3 and treated as described in (B). Total protein extracts were separated on 7.5\% SDS-PAGE gels. All samples shown in one panel are from the same blot, the dashed line was inserted to indicate an expected modification of the molecular weight of $\mathrm{NPH} 3$ due to truncations. The closed and open arrowheads indicate the positions of 'generally' phosphorylated and dephosphorylated NPH3 proteins, respectively. (D) In vivo interaction of RFP:NPH3 and phot1:GFP in transiently transformed N. benthamiana leaves adapted to darkness. Expression of transgenes was driven by the 35 S promoter. Microsomal proteins were immunoprecipitated using GFP beads and separated on $11 \%$ SDS-PAGE gels, followed by immunoblotting with anti-GFP and anti-RFP antibodies, respectively. (E) Model depicting the light-regime triggered changes in the phosphorylation status, subcellular localization and phototropic responsiveness of NPH3. BL-induced and phosphorylation-dependent (S744, blue) binding of 14-3-3 proteins releases NPH3 from the PM into the cytosol followed by condensate formation. Residues that are phosphorylated in darkness (yellow) and become dephosphorylated upon light treatment give rise to a shift in electrophoretic mobility ('general' phosphorylation status). Re-transfer to darkness reverts all BL-triggered processes, finally resulting in PM re-association. Cycling of NPH3 between the PM and the cytosol seems to be essential for proper function. Vice versa, NPH3 variants either constitutively attached to (red flash) or constitutively detached (red arrowhead) from the PM are non-functional.

\section{Supplementary Files}

This is a list of supplementary files associated with this preprint. Click to download.

- VideoS1.mov

- VideoS2.mov

- Videos3.mov

- VideoS4.mov

- VideoS5.mov

- VideoS6.mov

- VideoS7.mov 
- VideoS8.mov

- Videos9.mov 